\title{
Surgical or non-surgical treatment of traumatic skeletal fractures in adults: systematic review and meta-analysis of benefits and harms
}

\author{
Søren T. Skou ${ }^{1,2^{*}}$ (D), Carsten B. Juhl ${ }^{1,3}$, Kristoffer B. Hare ${ }^{2,4,5}$, L. Stefan Lohmander ${ }^{6}$ and Ewa M. Roos ${ }^{1}$
}

\begin{abstract}
Background: A comprehensive overview of treatments of common fractures is missing, although it would be important for shared decision-making in clinical practice. The aim was to determine benefits and harms of surgical compared to non-surgical treatments for traumatic skeletal fractures.

Methods: We searched Medline, Embase, CINAHL, Web of Science, and CENTRAL until November 2018, for randomized trials of surgical treatment in comparison with or in addition to non-surgical treatment of fractures in adults. For harms, only trials with patient enrollment in 2000 or later were included, while no time restriction was applied to benefits. Two reviewers independently assessed studies for inclusion, extracted data from full-text trials, and performed risk of bias assessment. Outcomes were self-reported pain, function, and quality of life, and serious adverse events (SAEs). Random effects model (Hedges' g) was used.

Results: Out of 28375 records screened, we included 61 trials and performed meta-analysis on 12 fracture types in 11 sites: calcaneus, clavicula, femur, humerus, malleolus, metacarpus, metatarsus, radius, rib, scaphoideum, and thoraco-lumbar spine. Seven other fracture types only had one trial available. For distal radius fractures, the standardized mean difference (SMD) was 0.31 ( $95 \% \mathrm{Cl} 0.10$ to $0.53, n=378$ participants) for function, favoring surgery, however, with greater risk of SAEs (RR = $3.10(1.42$ to 6.77$), n=436)$. For displaced intra-articular calcaneus fractures, SMD was 0.64 ( 0.13 to 1.16$)$ for function $(n=244)$ and 0.19 (0.01 to 0.36$)$ for quality of life $(n=506)$ favoring surgery. Surgery was associated with a smaller risk of SAE than non-surgical treatment for displaced midshaft clavicular fractures ( $R R=0.62$ (0.42 to 0.92$), n=1394)$. None of the other comparisons showed statistical significance differences and insufficient data existed for most of the common fracture types.

(Continued on next page)
\end{abstract}

\footnotetext{
* Correspondence: stskou@health.sdu.dk

${ }^{1}$ Research Unit for Musculoskeletal Function and Physiotherapy, Department of Sports Science and Clinical Biomechanics, University of Southern Denmark, Odense, Denmark

${ }^{2}$ Department of Physiotherapy and Occupational Therapy, Næstved-Slagelse-Ringsted Hospitals, Region Zealand, Slagelse, Denmark Full list of author information is available at the end of the article
}

(c) The Author(s). 2020, corrected publication 2021. Open Access This article is licensed under a Creative Commons Attribution 4.0 International License, which permits use, sharing, adaptation, distribution and reproduction in any medium or format, as long as you give appropriate credit to the original author(s) and the source, provide a link to the Creative Commons licence, and indicate if changes were made. The images or other third party material in this article are included in the article's Creative Commons licence, unless indicated otherwise in a credit line to the material. If material is not included in the article's Creative Commons licence and your intended use is not permitted by statutory regulation or exceeds the permitted use, you will need to obtain permission directly from the copyright holder. To view a copy of this licence, visit http://creativecommons.org/ licenses/by/4.0/. The Creative Commons Public Domain Dedication waiver (http://creativecommons.org/publicdomain/zero/1. 0/) applies to the data made available in this article, unless otherwise stated in a credit line to the data. 
(Continued from previous page)

Conclusions: Of 12 fracture types with more than one trial, only two demonstrated a difference in favor of surgery (distal radius fractures and displaced intra-articular calcaneus fractures), one of which demonstrated a greater risk of harms in the surgical group (distal radius fractures). Our results highlight the current paucity of high-quality randomized trials for common fracture types and a considerable heterogeneity and risk of bias in several of the available trials.

\section{Systematic review registration: PROSPERO CRD42015020805}

Keywords: Systematic review, Fracture, Therapeutics, Orthopedics, Randomized, controlled trial

\section{Background}

Fractures are an important public health burden. The agestandardized annual fracture incidence in England has been reported to be as high as $3.6 \%$ [1] with great variation dependent on how and from which population it is estimated [1-3]. Years lived with disability due to fractures are estimated to be around 22 million, most of which are long-term disability [4], and the total UK annual hospital costs associated with incident hip fractures in older adults alone are around $£ 1.1$ billion [5].

Surgery is the preferred treatment of most displaced fractures, but evidence from recent years suggests that non-surgical treatment might serve as an effective alternative for selected fractures, potentially associated with fewer adverse events and lower costs [6-9].

Fractures of the clavicula, humerus, radius, ulna, metacarpals, femur, and ankle are some of the most common fractures [2, 3]. However, a comprehensive overview of the benefits (e.g., improvements in pain, function, and quality of life) and harms (e.g., serious adverse events) of surgical and non-surgical treatment of these and other fractures is missing. A better understanding of the benefits and harms of these treatments for each of the most common fractures separately would serve as an important basis for shared decision-making about treatment of fractures in clinical practice.

We therefore aimed in this systematic review and meta-analysis to determine the benefits and harms of surgical compared with non-surgical treatments for acute, traumatic, skeletal fractures in adults. We extend existing knowledge [6-9] by including more recent trials and by including and analyzing outcomes on patientreported pain, physical function, quality of life, and SAE on each type of fracture separately.

\section{Methods}

This report conforms to the PRISMA statement [10]. The study followed the published guidelines on systematic reviews from the Cochrane Collaboration [11] and it was pre-registered with PROSPERO (CRD42015020805). In the PROSPERO-registration, two systematic reviews are described, the other being a systematic review of surgical vs. non-surgical treatment of non-fracture musculoskeletal conditions, which will be reported in a subsequent publication.

\section{Search strategy}

Two authors (STS + CBT) searched MEDLINE via PubMed, EMBASE via Ovid, CINAHL (including preCINAHL) via EBSCO, Web of Science via Web of Knowledge and CENTRAL, all up to 5 November 2018. We included trials reported in English, German, Danish, Swedish, and Norwegian (i.e., languages that the authors understand). For SAEs, only trials enrolling patients from 2000 were included due to the increasing quality of surgery and anesthesia and with the expectation of improved reporting of SAEs following the CONSORT statement published in 1996 and updated in 2001. No time restriction was applied for benefits. The search strategies were adjusted according to the specifications of the individual database (see Additional file S1). Reference lists of included articles and the most recent systematic reviews were reviewed to identify additional trials.

\section{Trial selection}

Two authors (STS + CBJ) independently assessed titles/ abstracts for trial eligibility using a priori selection criteria. The full text was retrieved if found eligible by at least one reviewer. The same authors independently evaluated eligibility of the retrieved full-text trials. Consensus was reached by discussion.

We included randomized trials conducted in any setting evaluating the effect of surgical treatment in comparison or in addition to non-surgical treatment of traumatic fractures in adults (mean age of trial participants 18+) with data on patient-reported pain, physical function, quality of life or SAEs. If any of these outcomes were reported, with data available that could be used in a meta-analysis, the trial was included. Surgery was pre-defined as any procedure that both changes the anatomy and requires a skin incision or use of an endoscopic technique [12], while non-surgical treatment was defined as all non-surgical treatments and placebo treatments.

Trials investigating the effects of drug substances used perioperatively, vertebroplasty, and kyphoplasty, cancer- 
related fractures, and jaw fractures were excluded. Conference abstracts were also excluded.

\section{Outcomes}

Our pre-defined outcomes of interest for benefit were patient-reported pain, physical function, and quality of life, and SAEs for harm. If more than one outcome was available for patient-reported pain, physical function, and quality of life, multidimensional outcomes were preferred before unidimensional outcomes. For unidimensional pain, pain intensity in the activity was preferred over pain intensity in rest. We pre-defined SAEs using the U.S. Food and Drug Administration definition, as all adverse events having the potential to significantly compromise the clinical outcome, result in significant disability or incapacity, requiring inpatient or outpatient hospital care, and those considered to prolong hospital care, to be life-threatening, or to result in death [13]. Non-unions were considered as SAE, while mal-unions were only considered as SAE if this resulted in additional treatment or significant disability or pain. Minor additional surgery such as removal of Kirschner wires was not considered an SAE, if they were part of normal clinical practice following the specific surgical procedure. Crossovers from non-surgical to surgical treatment were not considered an SAE unless caused by an SAE.

\section{Data extraction}

A customized data extraction form was developed for the outcomes, and two authors (STS + CBJ) independently extracted data. We preferred data from the 12 months follow-up of the trials, as this is a very common primary endpoint in trials of orthopedic surgery and as benefits from surgical and non-surgical treatment are expected to be stable at that time point. If data was not available from a 12-month follow-up, data from the follow-up closest to 12 months was used. We extracted the number of patients randomized to each treatment, age, sex, study location (country), pain, and BMI at baseline, fracture type, surgical and non-surgical intervention, follow-up time, number of patients not undergoing surgery in the surgical group, number of crossover to surgical treatment, number of patients analyzed, mean effect and SD, deaths and SAEs during follow-up and types of SAEs. If SAEs, deaths, or crossover were not mentioned, it was considered as if it had not occurred.

\section{Risk of bias assessment}

Risk of bias was assessed using the Risk of Bias 2.0 tool from the Cochrane Collaboration on trials with results on benefits [14]. Two authors (STS + CBJ) independently assessed if each of the following five domains was associated with low risk of bias, some concerns or high risk of bias: (1) bias arising from the randomization process, (2) bias due to deviations from intended interventions, (3) bias due to missing outcome data, (4) bias in measurement of the outcome, (5) bias in selection of the reported result. If four or five of the individual domains were found to be associated with some concerns of risk of bias, or if one of them was associated with a high risk of bias, the overall risk of bias was rated as high risk.

For SAEs (including death) trial quality was assessed independently on trials with results on SAEs by two authors (STS + CBJ) using the 15-point McMaster tool for assessing quality of harms assessment and reporting in study reports (McHarm) [15]. A score greater than 9 was considered a high score and indicative of low risk of bias.

Any discrepancies in the assessment of trial quality were resolved by discussion.

\section{Data synthesis and statistical methods}

The benefits of surgery were estimated using metaanalyses as the standardized mean difference (SMD) allowing for pooling the various outcomes assessed in the individual trials. The SMD was estimated as the difference in mean at follow-up in the intervention and control groups divided by the pooled SD. If the SD was not available it was estimated from the standard error, confidence interval, or the $P$ value, as recommended in the Cochrane Handbook [11]. If necessary, means and measures of dispersion were estimated from figures in the included trials. If only SD of the baseline score and SD of the change score were available, these were used for estimating SD of the final score [11]. SMD was adjusted to Hedges' g, as Cohen's d overestimate the effect in small studies. The SMD was interpreted clinically as originally proposed by Cohen [16], i.e., a SMD of 0.2 was small, a SMD of 0.5 was moderate, and a SMD of 0.8 was large. Heterogeneity was estimated as between-study variance $\left(\mathrm{tau}^{2}\right)$ and $I$-squared measuring the proportion of variation (i.e., inconsistency) in the combined estimates due to between-study variance. When $I$-squared is $0 \%$, no inconsistency is seen between results of individual trials and inconsistency is maximal when $I$-squared is $100 \%$.

SAEs were calculated as relative risk (RR). In order to handle null findings in either intervention or control group, Battaglias code was imputed. Battaglias code imputes one event distributed according to the numbers in the intervention and control group. The analyses of deaths followed the same approach. Results of individual studies were summed using a random-effects model meta-analysis for studies with relevant data on any of the outcomes, separated based on fracture type, body site, and outcome. While at least two studies were required to conduct meta-analyses on the different fracture types, all studies adhering to the eligibility criteria were included in the systematic review. 
A $p$ value less than 0.05 (two-sided) was considered significant. Analyses were carried out in Stata 15 (StataCorp, College Station, TX, USA).

\section{Results}

\section{Description of included trials}

The literature search revealed 41,186 hits and 59 were identified from other sources (i.e., references in systematic reviews and in included studies). After removing duplicates, we screened 28,375 titles and abstracts, which led to the retrieval of 192 full texts. After screening full texts, we ended up with 61 trials (in 62 publications) with relevant data available on either patient-reported pain, function, quality of life, and/or SAEs (Fig. 1). These trials were spread across 19 fracture types at 13 body sites: calcaneal (displaced intra-articular), clavicula (displaced midshaft, other), femur (Pipkin type), humerus (proximal, shaft), malleolar (trimalleolar, unstable (unibi- or trimalleolar), stable lateral malleolar, other), metacarpal (5th), metatarsal (5th), radius (distal), rib (flail chest), scaphoid (waist), tibia (shaft), thoraco-lumbar spine (traumatic), ulnar (olecranon and shaft) fractures.

Out of the 61 eligible trials ( $n=6021$ patients), 31 had data on pain $(n=2605), 45$ on function $(n=3735), 18$ on quality of life $(n=2306)$, 44 on SAEs $(n=3953)$, and 44 on deaths $(n=4145)$. Displaced midshaft clavicula $(n$

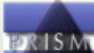

PRISMA 2009 Flow Diagram

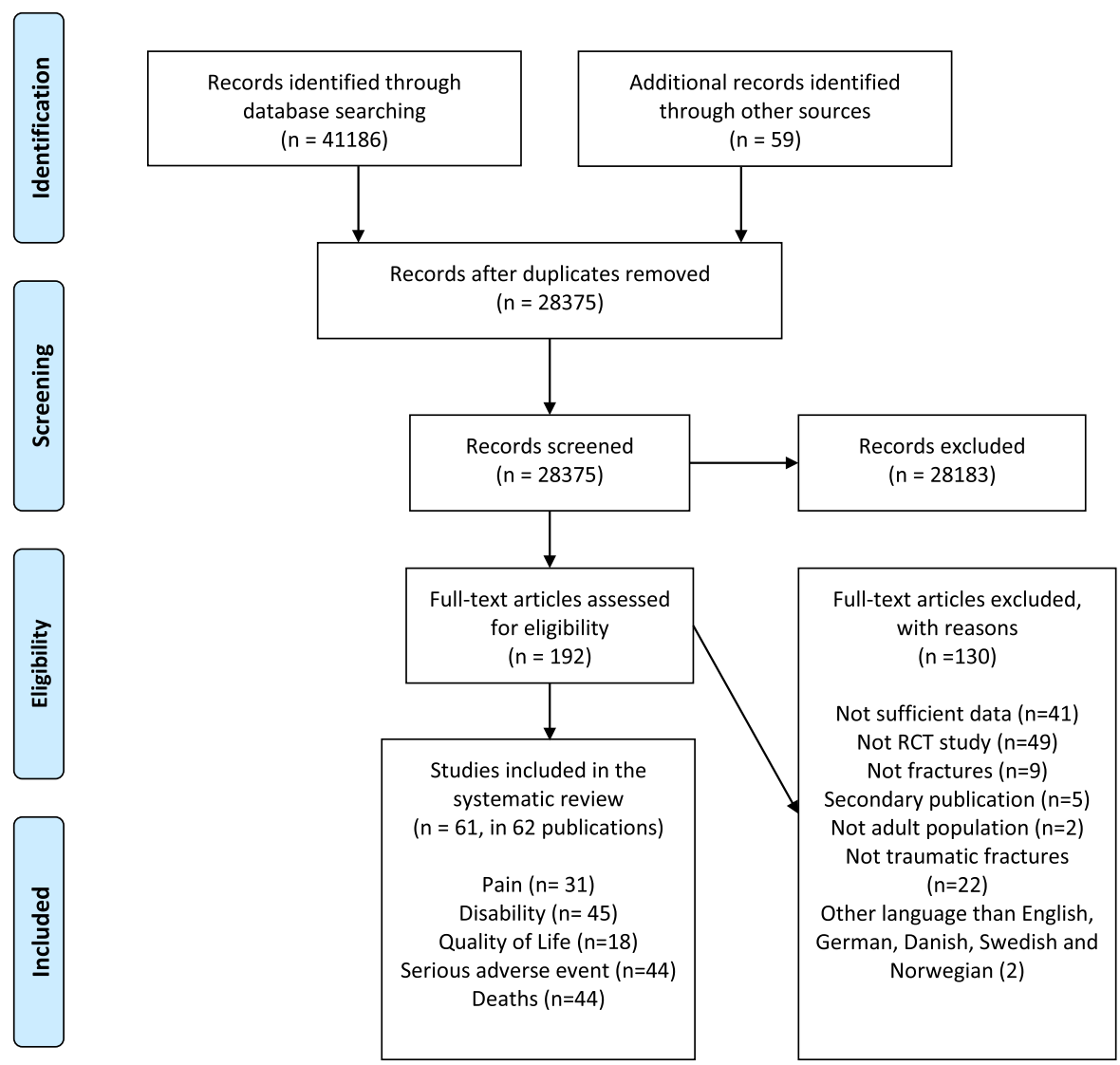

From: Moher D, Liberati A, Tetzlaff J, Altman DG, The PRISMA Group (2009). Preferred Reporting /tems for Systematic Reviews and MetaAnalyses: The PRISMA Statement. PLoS Med 6(7): e1000097. doi:10.1371/journal.pmed1000097 
Table 1 Summary of included trials of surgical and non-surgical treatment of fractures

\begin{tabular}{|c|c|c|c|c|c|c|c|c|}
\hline Fracture type & $\begin{array}{l}\text { Author, year, } \\
\text { country }\end{array}$ & $\begin{array}{l}\text { Age, } \% \\
\text { female }\end{array}$ & $\begin{array}{l}\text { Surgical } \\
\text { treatment } \\
(n)\end{array}$ & $\begin{array}{l}\text { Did not undergo } \\
\text { surgery after } \\
\text { randomization }(n)\end{array}$ & $\begin{array}{l}\text { Non- } \\
\text { surgical } \\
\text { treatment } \\
(n)\end{array}$ & $\begin{array}{l}\text { Received surgical } \\
\text { treatment in control } \\
\text { group }(n)\end{array}$ & $\begin{array}{l}\text { Benefit/ } \\
\text { harm } \\
\text { outcomes }\end{array}$ & $\begin{array}{l}\text { Follow-up } \\
\text { time } \\
\text { (month) }\end{array}$ \\
\hline \multirow[t]{6}{*}{$\begin{array}{l}\text { Calcaneal, } \\
\text { displaced } \\
\text { intraarticular }\end{array}$} & $\begin{array}{l}\text { Agren, 2013, } \\
\text { Sweden [17] }\end{array}$ & $\begin{array}{l}48.5 \\
\text { years, } \\
28.0 \%\end{array}$ & 42 & 0 & 40 & 0 & $\begin{array}{l}\text { Pain, } \\
\text { disability, } \\
\text { QoL }\end{array}$ & 12 months \\
\hline & $\begin{array}{l}\text { Buckley, } 2002[18] \\
\text { and O'Brien, Canada } \\
{[19]}\end{array}$ & $\begin{array}{l}40.0 \\
\text { years, } \\
10.3 \%\end{array}$ & 206 & 0 & 218 & 0 & Pain, QoL & 24 months \\
\hline & Griffin, 2014, UK [20] & $\begin{array}{l}46.4 \\
\text { years, } \\
15.9 \%\end{array}$ & 68 & 5 & 75 & 3 & $\begin{array}{l}\text { Disability, } \\
\text { QoL SAE }\end{array}$ & 24 months \\
\hline & $\begin{array}{l}\text { Ibrahim, 2007, UK } \\
\text { [21] }\end{array}$ & $\begin{array}{l}48.5 \\
\text { years, } \\
19.2 \%\end{array}$ & 25 & 0 & 31 & 0 & $\begin{array}{l}\text { Pain, } \\
\text { disability }\end{array}$ & $\begin{array}{l}180 \\
\text { months }\end{array}$ \\
\hline & $\begin{array}{l}\text { Nouraei, 2011, Iran } \\
\text { [22] }\end{array}$ & $\begin{array}{l}49 \\
\text { years, }\end{array}$ & 36 & 0 & 36 & 0 & Pain & 6 months \\
\hline & $\begin{array}{l}\text { Thordarson, 1996, } \\
\text { USA [23] }\end{array}$ & $\begin{array}{l}35.4 \\
\text { years, } \\
19.2 \%\end{array}$ & 16 & 0 & 14 & 0 & Disability & 16 months \\
\hline \multirow[t]{13}{*}{$\begin{array}{l}\text { Clavicular, } \\
\text { displaced } \\
\text { midshaft }\end{array}$} & $\begin{array}{l}\text { Ahrens, 2017, UK } \\
{[24]}\end{array}$ & $\begin{array}{l}36.2 \\
\text { years, } \\
13.6 \%\end{array}$ & 143 & 11 & 131 & 16 & $\begin{array}{l}\text { Disability, } \\
\text { SAE }\end{array}$ & 9 months \\
\hline & $\begin{array}{l}\text { Chen, 2011c, China } \\
\text { [25] }\end{array}$ & $\begin{array}{l}37.7 \\
\text { years, } \\
46.7 \%\end{array}$ & 30 & 0 & 30 & 0 & $\begin{array}{l}\text { Disability, } \\
\text { SAE }\end{array}$ & 15 months \\
\hline & $\begin{array}{l}\text { Judd, 2009, USA } \\
\text { [26] }\end{array}$ & $\begin{array}{l}26.5 \\
\text { years, } \\
87.7 \%\end{array}$ & 29 & 0 & 28 & 0 & $\begin{array}{l}\text { Disability, } \\
\text { SAE }\end{array}$ & 12 months \\
\hline & $\begin{array}{l}\text { Koch, 2008, } \\
\text { Germany [27] }\end{array}$ & $\begin{array}{l}35.4 \\
\text { years, } \\
33.8 \%\end{array}$ & 35 & 0 & 33 & 0 & Pain, SAE & 1 month \\
\hline & $\begin{array}{l}\text { Kumar, 2018, India } \\
{[28]}\end{array}$ & N/A & 40 & 0 & 40 & 0 & SAE & 12 months \\
\hline & $\begin{array}{l}\text { McKee, 2007, } \\
\text { Canada [29] }\end{array}$ & $\begin{array}{l}33.5 \\
\text { years, } \\
21.6 \%\end{array}$ & 66 & 1 & 64 & 1 & $\begin{array}{l}\text { Disability, } \\
\text { SAE }\end{array}$ & 12 months \\
\hline & $\begin{array}{l}\text { Melean, 2015, Chile } \\
\text { [30] }\end{array}$ & $\begin{array}{l}37.6 \\
\text { years, }\end{array}$ & 34 & 0 & 38 & 4 & SAE & 4 months \\
\hline & $\begin{array}{l}\text { Mirzatolooei, 2011, } \\
\text { Iran [31] }\end{array}$ & $\begin{array}{l}35.7 \\
\text { years, } \\
18.0 \%\end{array}$ & 29 & 3 & 31 & 0 & $\begin{array}{l}\text { Disability, } \\
\text { SAE }\end{array}$ & 12 months \\
\hline & $\begin{array}{l}\text { Qvist, 2018, } \\
\text { Denmark [32] }\end{array}$ & $\begin{array}{l}39.5 \\
\text { years, } \\
18.5 \%\end{array}$ & 74 & 1 & 66 & 9 & $\begin{array}{l}\text { Disability, } \\
\text { SAE }\end{array}$ & 12 months \\
\hline & $\begin{array}{l}\text { Robinson, 2013, UK } \\
\text { [33] }\end{array}$ & $\begin{array}{l}32.4 \\
\text { years, } \\
12.5 \%\end{array}$ & 95 & 0 & 92 & 13 & $\begin{array}{l}\text { Disability, } \\
\text { QoL, SAE }\end{array}$ & 12 months \\
\hline & $\begin{array}{l}\text { Smekal, 2009, } \\
\text { Austria [34] }\end{array}$ & $\begin{array}{l}37.7 \\
\text { years, } \\
13.3 \%\end{array}$ & 33 & 0 & 32 & 3 & $\begin{array}{l}\text { Disability, } \\
\text { SAE }\end{array}$ & 6 months \\
\hline & $\begin{array}{l}\text { Tamaoki, 2017, } \\
\text { Brazil [35] }\end{array}$ & $\begin{array}{l}32.5 \\
\text { years, } \\
14.5 \%\end{array}$ & 59 & 0 & 56 & 2 & $\begin{array}{l}\text { Pain, } \\
\text { disability, } \\
\text { SAE }\end{array}$ & 12 months \\
\hline & $\begin{array}{l}\text { Virtanen, 2012, } \\
\text { Finland [36] }\end{array}$ & $\begin{array}{l}36.7 \\
\text { years, } \\
13.3 \%\end{array}$ & 28 & 0 & 31 & 1 & $\begin{array}{l}\text { Pain, } \\
\text { disability, } \\
\text { SAE }\end{array}$ & 12 months \\
\hline
\end{tabular}


Table 1 Summary of included trials of surgical and non-surgical treatment of fractures (Continued)

\begin{tabular}{|c|c|c|c|c|c|c|c|c|}
\hline Fracture type & $\begin{array}{l}\text { Author, year, } \\
\text { country }\end{array}$ & $\begin{array}{l}\text { Age, } \% \\
\text { female }\end{array}$ & $\begin{array}{l}\text { Surgical } \\
\text { treatment } \\
(n)\end{array}$ & $\begin{array}{l}\text { Did not undergo } \\
\text { surgery after } \\
\text { randomization }(n)\end{array}$ & $\begin{array}{l}\text { Non- } \\
\text { surgical } \\
\text { treatment } \\
(n)\end{array}$ & $\begin{array}{l}\text { Received surgical } \\
\text { treatment in control } \\
\text { group }(n)\end{array}$ & $\begin{array}{l}\text { Benefit/ } \\
\text { harm } \\
\text { outcomes }\end{array}$ & $\begin{array}{l}\text { Follow-up } \\
\text { time } \\
\text { (month) }\end{array}$ \\
\hline & $\begin{array}{l}\text { Woltz, 2017, } \\
\text { Netherlands [37] }\end{array}$ & $\begin{array}{l}37.8 \\
\text { years, } \\
8.8 \%\end{array}$ & 86 & 0 & 62 & 12 & $\begin{array}{l}\text { Disability, } \\
\text { QoL, SAE }\end{array}$ & 12 months \\
\hline \multirow[t]{2}{*}{ Clavicular, other } & $\begin{array}{l}\text { Dugar, 2013, India } \\
\text { [38] }\end{array}$ & N/A & 15 & 0 & 15 & 0 & SAE & 12 months \\
\hline & $\begin{array}{l}\text { Yadav, 2015, India } \\
\text { [39] }\end{array}$ & $\begin{array}{l}33.1 \\
\text { years, } \\
20.0 \%\end{array}$ & 13 & 0 & 12 & 0 & SAE & 3 months \\
\hline \multirow[t]{2}{*}{ Femoral, caput } & $\begin{array}{l}\text { Chen, 2011a, China } \\
\text { [40] }\end{array}$ & $\begin{array}{l}37.5 \\
\text { years, } \\
18.8 \%\end{array}$ & 8 & 0 & 8 & 0 & SAE & 38 months \\
\hline & $\begin{array}{l}\text { Chen, } 2011 \text { b, China } \\
\text { [41] }\end{array}$ & $\begin{array}{l}38.7 \\
\text { years, } \\
29.2 \%\end{array}$ & 12 & 0 & 10 & 2 & SAE & 39 months \\
\hline Humeral shaft & $\begin{array}{l}\text { Matsunaga, 2017, } \\
\text { Brazil [42] }\end{array}$ & $\begin{array}{l}38.7 \\
\text { years, } \\
33.6 \%\end{array}$ & 52 & 0 & 10 & 48 & $\begin{array}{l}\text { Pain, } \\
\text { disability, } \\
\text { QoL, SAE }\end{array}$ & 12 months \\
\hline \multirow[t]{6}{*}{$\begin{array}{l}\text { Humeral, } \\
\text { proximal }\end{array}$} & $\begin{array}{l}\text { Boons, 2012, } \\
\text { Netherlands [43] }\end{array}$ & $\begin{array}{l}78.2 \\
\text { years, } \\
94.0 \%\end{array}$ & 25 & 0 & 25 & 0 & $\begin{array}{l}\text { Pain, } \\
\text { disability, } \\
\text { SAE }\end{array}$ & 12 months \\
\hline & $\begin{array}{l}\text { Fjalestad, 2014, } \\
\text { Norway [44] }\end{array}$ & $\begin{array}{l}72.6 \\
\text { years, } \\
88.0 \%\end{array}$ & 25 & 0 & 24 & 1 & $\begin{array}{l}\text { Disability, } \\
\text { QoL SAE }\end{array}$ & 12 months \\
\hline & $\begin{array}{l}\text { Olerud, 2011a, } \\
\text { Sweden [45] }\end{array}$ & $\begin{array}{l}73.9 \\
\text { years, } \\
81.4 \%\end{array}$ & 27 & 0 & 27 & 1 & $\begin{array}{l}\text { Pain, } \\
\text { Disability, } \\
\text { QoL, SAE }\end{array}$ & 12 months \\
\hline & $\begin{array}{l}\text { Olerud, 2011b, } \\
\text { Sweden [46] }\end{array}$ & $\begin{array}{l}76.7 \\
\text { years, } \\
85.5 \%\end{array}$ & 30 & 0 & 30 & 0 & $\begin{array}{l}\text { Pain, } \\
\text { disability, } \\
\text { QoL, SAE }\end{array}$ & 12 months \\
\hline & $\begin{array}{l}\text { Rangan, 2015, UK } \\
{[47]}\end{array}$ & $\begin{array}{l}66.0 \\
\text { years, } \\
76.8 \%\end{array}$ & 109 & 16 & 112 & 13 & $\begin{array}{l}\text { Disability, } \\
\text { QoL, SAE }\end{array}$ & 12 months \\
\hline & $\begin{array}{l}\text { Zyto, 1997, Sweden } \\
\text { [48] }\end{array}$ & $\begin{array}{l}74.0 \\
\text { years, } \\
87.5 \%\end{array}$ & 20 & 0 & 20 & 5 & $\begin{array}{l}\text { Pain, } \\
\text { disability }\end{array}$ & 50 months \\
\hline \multirow[t]{2}{*}{ Malleolar, other } & $\begin{array}{l}\text { Makwana, 2001, UK } \\
\text { [49] }\end{array}$ & $\begin{array}{l}66.9 \\
\text { years, } \\
72.1 \%\end{array}$ & 22 & 0 & 14 & 8 & $\begin{array}{l}\text { Pain, } \\
\text { disability }\end{array}$ & 27 months \\
\hline & Willet, 2016, UK [50] & $\begin{array}{l}70.6 \\
\text { years, } \\
74.2 \%\end{array}$ & 302 & 7 & 277 & 34 & $\begin{array}{l}\text { Pain, } \\
\text { disability, } \\
\text { QoL, SAE }\end{array}$ & 6 months \\
\hline Malleolar, stable & $\begin{array}{l}\text { Mittal, 2017, } \\
\text { Australia and New } \\
\text { Zealand [51] }\end{array}$ & $\begin{array}{l}39.0 \\
\text { years, } \\
51.9 \%\end{array}$ & 72 & 8 & 78 & 2 & $\begin{array}{l}\text { Disability, } \\
\text { QoL, SAE }\end{array}$ & 12 months \\
\hline $\begin{array}{l}\text { Malleolar, } \\
\text { trimalleolar }\end{array}$ & $\begin{array}{l}\text { Salai, 2000, Israel } \\
\text { [52] }\end{array}$ & $\begin{array}{l}78.3 \\
\text { years, } \\
75.0 \%\end{array}$ & 46 & 0 & 8 & 30 & $\begin{array}{l}\text { Pain, } \\
\text { disability }\end{array}$ & 38 months \\
\hline $\begin{array}{l}\text { Malleolar, } \\
\text { unstable }\end{array}$ & $\begin{array}{l}\text { Sanders, 2012, } \\
\text { Canada [53] }\end{array}$ & $\begin{array}{l}41.0 \\
\text { years, } \\
49.4 \%\end{array}$ & 41 & 0 & 39 & 1 & $\begin{array}{l}\text { Disability, } \\
\text { QoL, SAE }\end{array}$ & 12 months \\
\hline $\begin{array}{l}\text { Metacarpal, 5th } \\
\text { metacarpal neck }\end{array}$ & $\begin{array}{l}\text { Sletten, 2015, } \\
\text { Norway [54 ] }\end{array}$ & $\begin{array}{l}27.0 \\
\text { years, }\end{array}$ & 38 & 4 & 43 & 0 & $\begin{array}{l}\text { Disability, } \\
\text { SAE }\end{array}$ & 12 months \\
\hline
\end{tabular}


Table 1 Summary of included trials of surgical and non-surgical treatment of fractures (Continued)

\begin{tabular}{|c|c|c|c|c|c|c|c|c|}
\hline Fracture type & $\begin{array}{l}\text { Author, year, } \\
\text { country }\end{array}$ & $\begin{array}{l}\text { Age, } \% \\
\text { female }\end{array}$ & $\begin{array}{l}\text { Surgical } \\
\text { treatment } \\
(n)\end{array}$ & $\begin{array}{l}\text { Did not undergo } \\
\text { surgery after } \\
\text { randomization }(n)\end{array}$ & $\begin{array}{l}\text { Non- } \\
\text { surgical } \\
\text { treatment } \\
(n)\end{array}$ & $\begin{array}{l}\text { Received surgical } \\
\text { treatment in control } \\
\text { group }(n)\end{array}$ & $\begin{array}{l}\text { Benefit/ } \\
\text { harm } \\
\text { outcomes }\end{array}$ & $\begin{array}{l}\text { Follow-up } \\
\text { time } \\
\text { (month) }\end{array}$ \\
\hline & $\begin{array}{l}\text { Strub, 2010, } \\
\text { Switzerland [55] }\end{array}$ & $\begin{array}{l}30.0 \\
\text { years, } \\
5.0 \%\end{array}$ & 20 & 0 & 20 & 0 & SAE & 12 months \\
\hline \multirow[t]{2}{*}{$\begin{array}{l}\text { Metatarsal, 5th } \\
\text { metatarsal neck }\end{array}$} & $\begin{array}{l}\text { Lee, 2016, South } \\
\text { Korea [56] }\end{array}$ & $\begin{array}{l}41.7 \\
\text { years, } \\
55.2 \%\end{array}$ & 9 & 0 & 9 & 0 & Pain & 2 months \\
\hline & $\begin{array}{l}\text { Wu, 2018, China } \\
\text { [57] }\end{array}$ & $\begin{array}{l}27.1 \\
\text { years, } \\
36.6 \%\end{array}$ & 23 & 0 & 22 & 1 & Pain, SAE & 12 months \\
\hline \multirow[t]{8}{*}{ Radial, distal } & $\begin{array}{l}\text { Abbaszadegan, } \\
\text { 1990, Sweden [58] }\end{array}$ & $\begin{array}{l}63.0 \\
\text { years, } \\
76.6 \%\end{array}$ & 23 & 0 & 24 & 0 & $\begin{array}{l}\text { Pain, } \\
\text { disability }\end{array}$ & 12 months \\
\hline & $\begin{array}{l}\text { Arora, 2011, Austria } \\
\text { [59] }\end{array}$ & $\begin{array}{l}76.7 \\
\text { years, } \\
75.3 \%\end{array}$ & 45 & 0 & 45 & 0 & $\begin{array}{l}\text { Pain, } \\
\text { disability, } \\
\text { SAE }\end{array}$ & 12 months \\
\hline & $\begin{array}{l}\text { Azzopardi, 2005, UK } \\
\text { [60] }\end{array}$ & $\begin{array}{l}71.5 \\
\text { years, } \\
88.9 \%\end{array}$ & 30 & 0 & 27 & 0 & $\begin{array}{l}\text { Pain, } \\
\text { disability, } \\
\text { QoL, SAE }\end{array}$ & 12 months \\
\hline & $\begin{array}{l}\text { Földhazy, 2010, } \\
\text { Sweden [61] }\end{array}$ & $\begin{array}{l}71.6 \\
\text { years, } \\
89.8 \%\end{array}$ & 28 & 0 & 31 & 0 & $\begin{array}{l}\text { Pain, } \\
\text { disability, } \\
\text { SAE }\end{array}$ & 12 months \\
\hline & $\begin{array}{l}\text { Kreder, 2006, } \\
\text { Canada and USA } \\
\text { [62] }\end{array}$ & $\begin{array}{l}52.9 \\
\text { years, } \\
65.5 \%\end{array}$ & 54 & 0 & 54 & 5 & $\begin{array}{l}\text { Pain, } \\
\text { disability }\end{array}$ & 12 months \\
\hline & $\begin{array}{l}\text { Mardani Kivi, 2011, } \\
\text { Iran [63] }\end{array}$ & $\begin{array}{l}50.8 \\
\text { years, } \\
13.0 \%\end{array}$ & 99 & 0 & 93 & 6 & SAE & 3 months \\
\hline & $\begin{array}{l}\text { McQueen, 2008, UK } \\
\text { [64] }\end{array}$ & $\begin{array}{l}29.4 \\
\text { years, } \\
16.7 \%\end{array}$ & 30 & 0 & 30 & 0 & SAE & 12 months \\
\hline & $\begin{array}{l}\text { Wong, 2010, Hong } \\
\text { Kong [65] }\end{array}$ & $\begin{array}{l}70.5 \\
\text { years, } \\
81.7 \%\end{array}$ & 31 & 0 & 31 & 0 & $\begin{array}{l}\text { Pain, } \\
\text { disability, } \\
\text { QoL, SAE }\end{array}$ & 12 months \\
\hline Rib, flail chest & $\begin{array}{l}\text { Marasco, 2013, } \\
\text { Australia [66] }\end{array}$ & $\begin{array}{l}58.5 \\
\text { years, } \\
13.0 \%\end{array}$ & 22 & 1 & 23 & 0 & $\begin{array}{l}\text { Pain, } \\
\text { disability, } \\
\text { QoL, SAE }\end{array}$ & 6 months \\
\hline \multirow[t]{4}{*}{ Scaphoid, waist } & $\begin{array}{l}\text { Arora, 2007, Austria } \\
\text { [67] }\end{array}$ & $\begin{array}{l}33.0 \\
\text { years, } \\
27.3 \%\end{array}$ & 23 & 0 & 24 & 0 & $\begin{array}{l}\text { Pain, } \\
\text { disability, } \\
\text { SAE }\end{array}$ & 6 months \\
\hline & $\begin{array}{l}\text { Clementson, 2015, } \\
\text { Sweden [68] }\end{array}$ & $\begin{array}{l}31.4 \\
\text { years, } \\
18.4 \%\end{array}$ & 13 & 1 & 24 & 0 & $\begin{array}{l}\text { disability, } \\
\text { SAE }\end{array}$ & 12 months \\
\hline & Dias, 2005, UK [69] & $\begin{array}{l}29.5 \\
\text { years, } \\
10.2 \%\end{array}$ & 44 & 0 & 37 & 7 & Pain & 12 months \\
\hline & $\begin{array}{l}\text { Vinnars, 2008, } \\
\text { Sweden [70] }\end{array}$ & $\begin{array}{l}30.5 \\
\text { years, } \\
22.7 \%\end{array}$ & 40 & 3 & 41 & 1 & Disability & $\begin{array}{l}120 \\
\text { months }\end{array}$ \\
\hline \multirow[t]{2}{*}{$\begin{array}{l}\text { Thoraco-lumbal, } \\
\text { traumatic } \\
\text { compression }\end{array}$} & $\begin{array}{l}\text { Piazzolla, 2011, Italy } \\
\text { [71] }\end{array}$ & $\begin{array}{l}39.9 \\
\text { years, } \\
36.0 \%\end{array}$ & 24 & 0 & 26 & 0 & $\begin{array}{l}\text { Pain, } \\
\text { disability, } \\
\text { SAE }\end{array}$ & 12 months \\
\hline & $\begin{array}{l}\text { Shen, 2001, Taiwan } \\
\text { [72] }\end{array}$ & $\begin{array}{l}43.2 \\
\text { years, }\end{array}$ & 33 & 7 & 43 & 0 & $\begin{array}{l}\text { Pain, } \\
\text { disability }\end{array}$ & 12 months \\
\hline
\end{tabular}


Table 1 Summary of included trials of surgical and non-surgical treatment of fractures (Continued)

\begin{tabular}{|c|c|c|c|c|c|c|c|c|}
\hline Fracture type & $\begin{array}{l}\text { Author, year, } \\
\text { country }\end{array}$ & $\begin{array}{l}\text { Age, } \% \\
\text { female }\end{array}$ & $\begin{array}{l}\text { Surgical } \\
\text { treatment } \\
\text { (n) }\end{array}$ & $\begin{array}{l}\text { Did not undergo } \\
\text { surgery after } \\
\text { randomization (n) }\end{array}$ & $\begin{array}{l}\text { Non- } \\
\text { surgical } \\
\text { treatment } \\
\text { (n) }\end{array}$ & $\begin{array}{l}\text { Received surgical } \\
\text { treatment in control } \\
\text { group }(n)\end{array}$ & $\begin{array}{l}\text { Benefit/ } \\
\text { harm } \\
\text { outcomes }\end{array}$ & $\begin{array}{l}\text { Follow-up } \\
\text { time } \\
\text { (month) }\end{array}$ \\
\hline & $\begin{array}{l}\text { Siebenga, 2006, } \\
\text { Netherlands [73] }\end{array}$ & $\begin{array}{l}41.8 \\
\text { years, } \\
37.5 \%\end{array}$ & 18 & 0 & 16 & 0 & $\begin{array}{l}\text { Pain, } \\
\text { disability }\end{array}$ & 52 months \\
\hline & $\begin{array}{l}\text { Wood, 2003, USA } \\
\text { [74] }\end{array}$ & $\begin{array}{l}41.4 \\
\text { years, } \\
31.9 \%\end{array}$ & 26 & 0 & 26 & 1 & $\begin{array}{l}\text { Pain, } \\
\text { disability, } \\
\text { QoL }\end{array}$ & 46 months \\
\hline \multirow[t]{2}{*}{ Tibial shaft } & $\begin{array}{l}\text { Karladani, 2000, } \\
\text { Sweden [75] }\end{array}$ & $\begin{array}{l}39.0 \\
\text { years, } \\
32.1 \%\end{array}$ & 27 & 0 & 12 & 17 & $\begin{array}{l}\text { Pain, } \\
\text { Disability, } \\
\text { QoL }\end{array}$ & 12 months \\
\hline & $\begin{array}{l}\text { Granetzny, 2005, } \\
\text { Egypt [76] }\end{array}$ & $\begin{array}{l}38.2 \\
\text { years, } \\
22.5 \%\end{array}$ & 20 & 0 & 20 & 0 & SAE & 2 months \\
\hline Ulnar shaft & $\begin{array}{l}\text { Hussain, 2018, India } \\
\text { [77] }\end{array}$ & $\begin{array}{l}38.9 \\
\text { years, } \\
13.3 \%\end{array}$ & 20 & 0 & 17 & 3 & $\begin{array}{l}\text { Disability, } \\
\text { SAE }\end{array}$ & 12 months \\
\hline Ulnar, olecranon & $\begin{array}{l}\text { Duckworth, 2017, } \\
\text { UK [78] }\end{array}$ & $\begin{array}{l}82.9 \\
\text { years, } \\
89.5 \%\end{array}$ & 11 & 0 & 1 & 7 & $\begin{array}{l}\text { Disability, } \\
\text { SAE }\end{array}$ & 12 months \\
\hline
\end{tabular}

QoL quality of life; $S A E$ serious adverse events

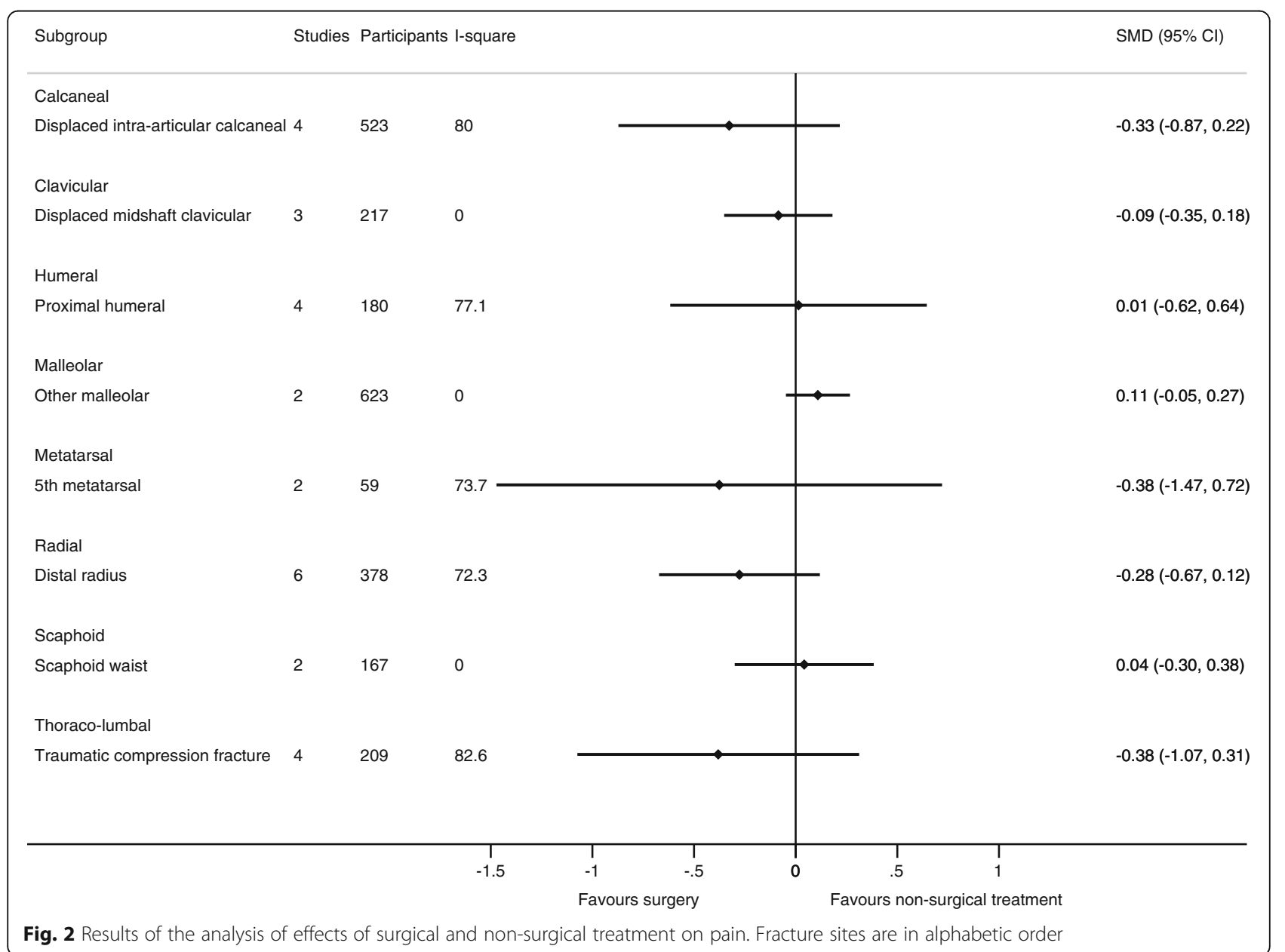


$=14$ trials), distal radius $(n=7)$, displaced intra-articular calcaneus $(n=6)$, scaphoid waist $(n=6)$, and proximal humerus $(n=6)$ fractures were the fractures most commonly investigated. Trials were carried out across 24 different countries, with the UK $(n=11)$, Sweden $(n=9)$, and the USA $(n=6)$ being the most common. Age and gender distribution varied depending on the fracture type. Table 1 presents the characteristics of the included trials.

As only one trial with relevant data was available for humeral shaft, malleolar (trimalleolar, unstable (uni- bior trimalleolar), stable lateral malleolar), tibia (shaft), and ulnar (olecranon and shaft) fractures, respectively, only 12 fracture types in 11 body sites were evaluated in meta-analyses. See Figs. 2, 3, 4, and 5 for the number of trials and patients included in the meta-analyses within each of the fracture types for each of the outcomes.

\section{Benefits}

\section{Synthesis of results}

The results of the meta-analytic syntheses for each of the fracture types separately are presented in Fig. 2 (pain), Fig. 3 (function), and in Fig. 4 (quality of life).
For 6 out of the 8 fracture types with available data on pain, function, and quality of life from at least two trials, no important differences in pain and function were demonstrated between surgical and non-surgical treatment. No studies included a placebo treatment.

For 2 fracture types, surgical treatment was associated with greater benefits. For distal radius fractures (6 trial $\mathrm{s}[58-62,65](n=378))$, the SMD was $0.31(0.10$ to 0.53$)$ for function. For displaced intra-articular calcaneus fractures $(4[17,20,21,23] / 3[17,18,20]$ trials $(n=244 /$ 506), SMD was 0.64 (0.13 to 1.16) for function, and 0.19 (0.01 to 0.36 ) for quality of life.

Additional file S2 presents the full forest plots for all comparisons.

One trial on trimalleolar ankle fractures $(n=65)$ [52] and one trial on tibial shaft fractures $(n=53)$ [75] also demonstrated a significant effect for function in favor of surgery.

\section{Risk of bias}

Table 2 presents the risk of bias assessment for the individual trials.

Overall, no trials were judged as low risk of bias and 17 out of 52 trials $[18,21,22,27,28,31,49,52,56,58$, $60,61,67,72,75,77,79]$ were associated with a high

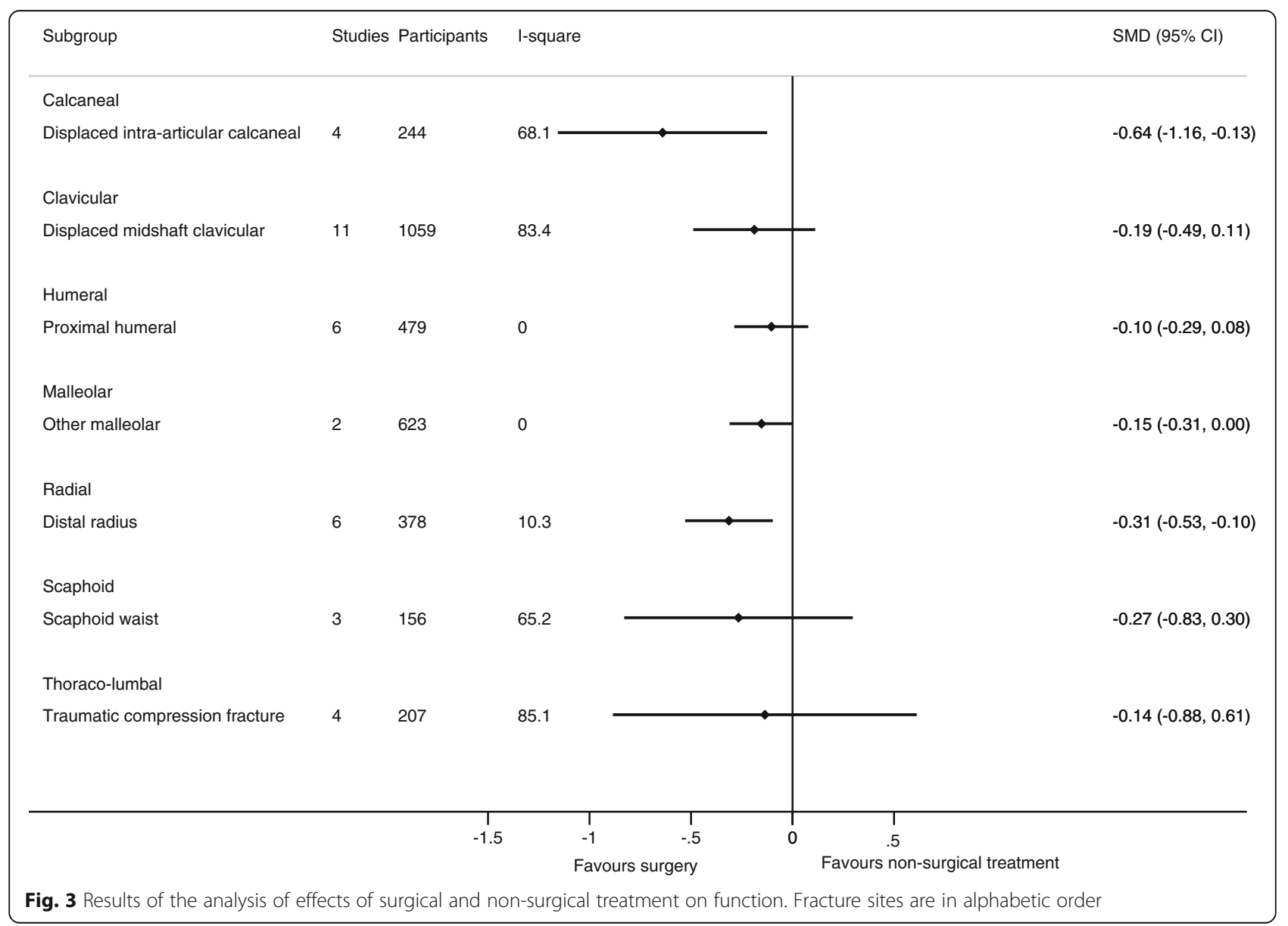




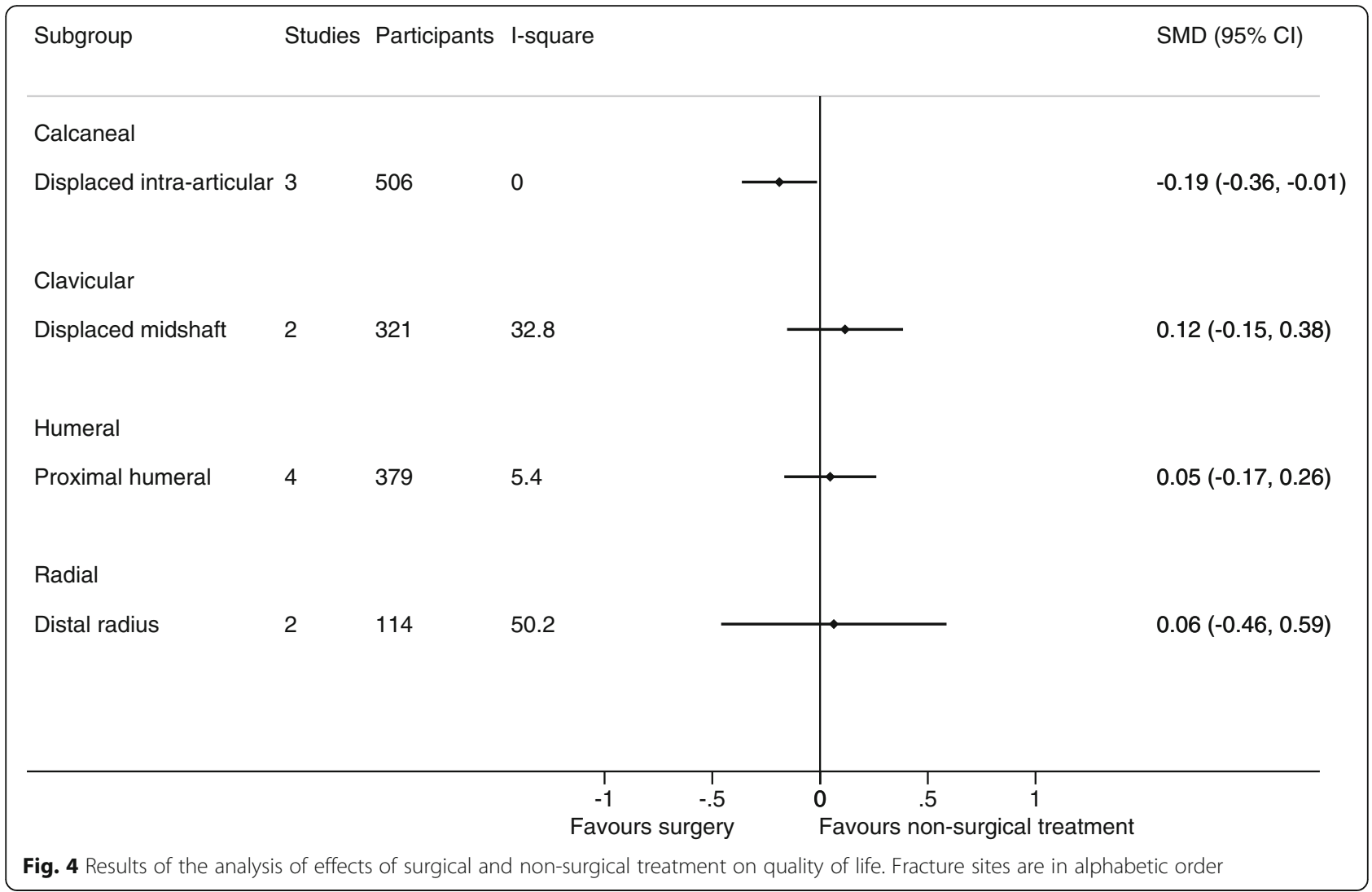

risk of bias, mainly due to the lack of possibility to blind patients and treatment providers, and lack of preregistration of the trial in a public trial registry before enrolment of the first patient.

\section{Harms}

\section{Synthesis of results}

The syntheses of the results are presented in Fig. 5 (SAEs), and in Additional file S2 (deaths and the full forest plot for SAEs).

For 6 out of the 8 fracture types with available data on SAEs from at least two trials, no differences were demonstrated between surgical and non-surgical treatment. For displaced midshaft clavicula fractures $(14$ trial $(n=1394))$ [24-37], surgery was associated with a smaller risk of SAEs than non-surgical treatment (RR $0.62(0.42$ to 0.92$)$ ). For distal radius fracture $(5$ trials $(\mathrm{n}=436))$ [59-61, 65, 80], surgery was associated with a greater risk of SAEs than non-surgical treatment (RR 3.10, 95\% CI 1.42 to 6.77).

One trial on unstable malleolar fractures $(n=592)$ [50] and one trial on humeral shaft fractures $(n=96)$ [42] demonstrated fewer SAEs in the surgical compared to the non-surgical group.

There were no differences between surgical and nonsurgical treatment in the risk of death for any of the fracture types.

\section{Risk of bias}

Additional file S3 presents the risk of bias assessment for the individual trials.

Overall, the risk of bias associated with the assessment and reporting of SAEs and death was moderate to high. Only two trials $[20,53]$ had a score greater than 9 indicating a low risk of bias.

\section{Discussion}

We found a difference in function in favor of surgery (moderate effect) for displaced intraarticular calcaneal fractures (however with large heterogeneity due to a small $(n=30)$, old study) and distal radial fractures (small effect), however, with increased risk of SAEs after surgery for radial fractures. No difference in effect was demonstrated for displaced midshaft clavicular fractures and proximal humeral fractures, scaphoid waist, and thoracolumbar traumatic compression fractures, while surgery for clavicular fractures was associated with reduced risk of SAE. Insufficient data existed for all other fracture types.

The large inconsistency and often missing reporting of SAEs and death in the included trials represent a limitation of our study. The lack of consensus in terms and definitions of complications after treatment of fractures calls for the development and validation of a core set of 


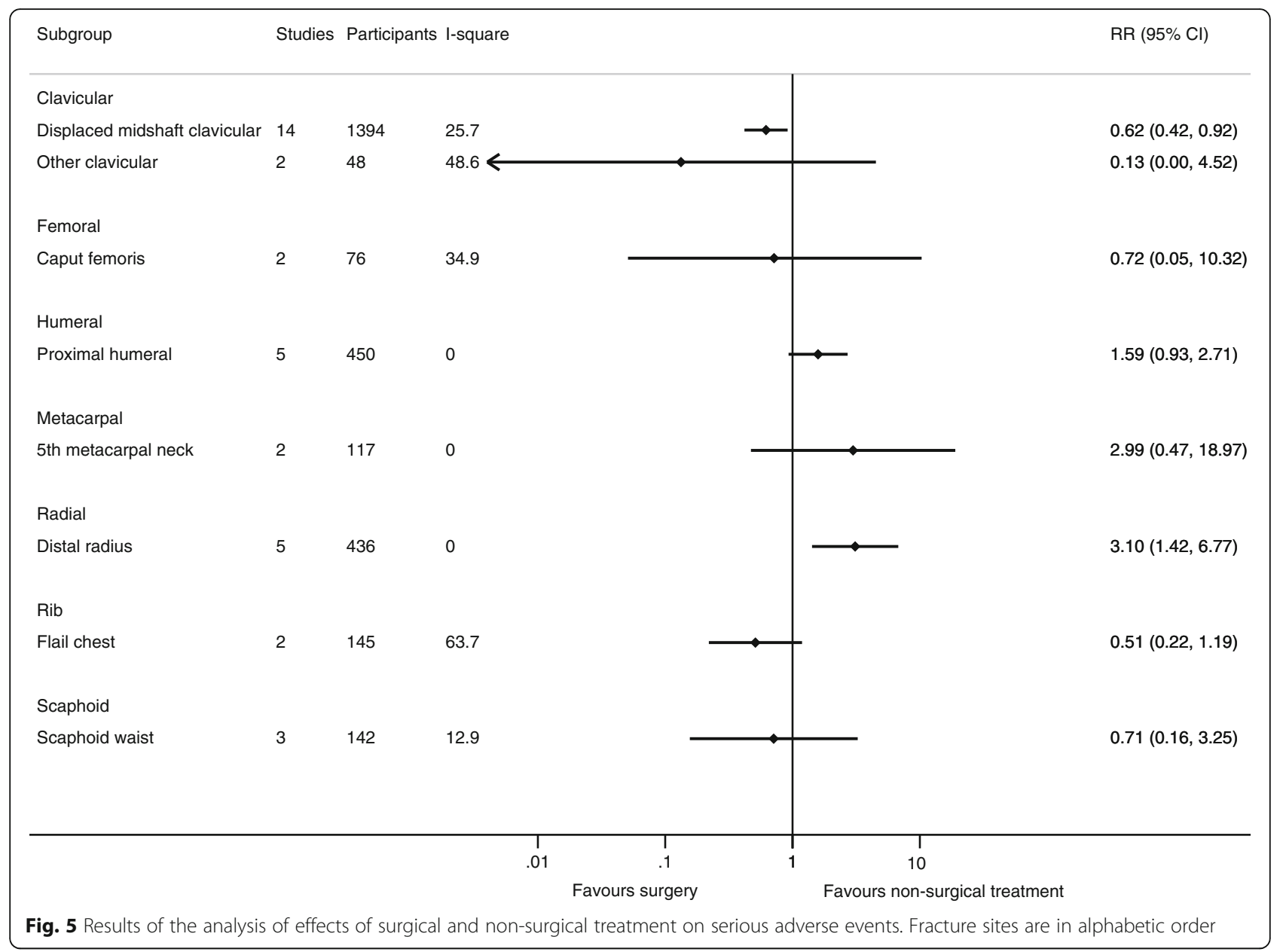

complications [81]. Another potential limitation of this study relates to our selection of outcomes, as 39 trials were excluded due to insufficient data. Some of the trials had selected composite scores of, e.g., pain and function or other outcomes like time to healing of the fracture, while others did not report data that could be included in meta-analyses, e.g., by reporting pain evaluated on a 5point Likert scale. For feasibility reasons, we excluded trials that were not in languages understood by any of the authors, which could be a potential bias. However, as only two trials were excluded based on this criterion, the expected impact on the results is considered minimal. Finally, from a clinical point of view, it is common to decide on whether to recommend surgery or not based not only on the fracture type, but also on patient characteristics such as age, work status, and symptom severity. In pragmatic trials, patients are more commonly included without accounting for patient characteristics, which thereby can potentially affect the generalizability of the results from the individual meta-analyses of this study [63].

Although our results could indicate that non-surgical treatment is as effective as surgical treatment for several traumatic fractures in adults, including displaced midshaft clavicular, proximal humeral, scaphoid waist, and thoracolumbar traumatic compression fractures, serious caveats relating to the number of patients studied, heterogeneity and study methodology question the confidence in such a suggestion. First, only $7 / 19$ fracture types had been scrutinized in at least 2 trials with at least 100 patients totally. Second, few and underpowered studies for some fracture types might be part of the explanation for our findings [82], as a previous study found a mean overall study power (1-beta) among 117 trials of traumatic skeletal fractures of 25\% [83]. Third, none of the included trials were associated with a low risk of bias for benefits, and only 2/44 (5\%) trials were associated with a low risk of bias for SAEs, confirming a previous study summarizing orthopedic trials [82]. In fact, $17 / 52(33 \%)$ of the trials with data on benefits were associated with a high risk of bias. Finally, the studied fracture types only represent selected types of fractures in selected types of patients. For some fractures (e.g., clavicular and stable lateral malleolar fractures), the natural history of healing without surgical treatment has a good prognosis [84-86]. However, in older persons with lower expectations of function with, e.g., a distal radius or malleolar fracture and more osteoporotic bone, the expected beneficial effect 
Table 2 Assessment of risk of bias of included trials of surgical and non-surgical treatment of fractures

\begin{tabular}{|c|c|c|c|c|c|c|}
\hline Author, year & $\begin{array}{l}\text { Randomization } \\
\text { process }\end{array}$ & $\begin{array}{l}\text { Deviations from intended } \\
\text { interventions }\end{array}$ & $\begin{array}{l}\text { Missing } \\
\text { outcome data }\end{array}$ & $\begin{array}{l}\text { Measurement of the } \\
\text { outcome }\end{array}$ & $\begin{array}{l}\text { Selection of the } \\
\text { reported result }\end{array}$ & $\begin{array}{l}\text { Overall } \\
\text { bias }\end{array}$ \\
\hline $\begin{array}{l}\text { Abbaszadegan, } \\
1990\end{array}$ & Some concern & Some concern & Low risk & Some concern & Some concern & High risk \\
\hline Agren, 2013 & Low risk & Low risk & Low risk & Some concern & Some concern & $\begin{array}{l}\text { Some } \\
\text { concern }\end{array}$ \\
\hline Ahrens, 2017 & Low risk & Some concern & Low risk & Some concern & Some concern & $\begin{array}{l}\text { Some } \\
\text { concern }\end{array}$ \\
\hline Arora, 2007 & Some concern & Low risk & Some concern & Some concern & Some concern & High risk \\
\hline Arora, 2011 & Low risk & Low risk & Low risk & Some concern & Some concern & $\begin{array}{l}\text { Some } \\
\text { concern }\end{array}$ \\
\hline Azzopardi, 2005 & Some concern & Low risk & Some concern & Some concern & Some concern & High risk \\
\hline Boons, 2012 & Low risk & Low risk & Low risk & Some concern & Some concern & $\begin{array}{l}\text { Some } \\
\text { concern }\end{array}$ \\
\hline Buckley, 2002 & Low risk & Low risk & High risk & Some concern & Some concern & High risk \\
\hline Chen, 2011c & Some concern & Low risk & Low risk & Some concern & Some concern & $\begin{array}{l}\text { Some } \\
\text { concern }\end{array}$ \\
\hline $\begin{array}{l}\text { Clementson, } \\
2015\end{array}$ & Low risk & Some concern & High risk & Some concern & Some concern & High risk \\
\hline Dias, 2005 & Low risk & Low risk & Some concern & Some concern & Some concern & $\begin{array}{l}\text { Some } \\
\text { concern }\end{array}$ \\
\hline $\begin{array}{l}\text { Duckworth, } \\
2017\end{array}$ & Low risk & Low risk & Some concern & Some concern & Some concern & $\begin{array}{l}\text { Some } \\
\text { concern }\end{array}$ \\
\hline Fjalestad, 2014 & Low risk & Low risk & Low risk & Some concern & Some concern & $\begin{array}{l}\text { Some } \\
\text { concern }\end{array}$ \\
\hline Földhazy, 2010 & Low risk & Some concern & Some concern & Some concern & Some concern & High risk \\
\hline Griffin, 2014 & Low risk & Low risk & Low risk & Some concern & Low risk & $\begin{array}{l}\text { Some } \\
\text { concern }\end{array}$ \\
\hline Hussain, 2017 & Some concern & Some concern & Some concern & Some concern & Some concern & High risk \\
\hline Ibrahim, 2007 & High risk & Some concern & High risk & Some concern & Some concern & High risk \\
\hline Judd, 2009 & Low risk & Low risk & Some concern & Some concern & Some concern & $\begin{array}{l}\text { Some } \\
\text { concern }\end{array}$ \\
\hline Karladani, 2000 & Some concern & High risk & Some concern & Some concern & Some concern & High risk \\
\hline Koch, 2008 & Some concern & Some concern & Some concern & Some concern & Some concern & High risk \\
\hline Kreder, 2006 & Low risk & Low risk & Some concern & Some concern & Some concern & $\begin{array}{l}\text { Some } \\
\text { concern }\end{array}$ \\
\hline Kumar, 2018 & High risk & Some concern & Some concern & Some concern & Some concern & High risk \\
\hline Lee, 2016 & Some concern & Some concern & Low risk & Some concern & Some concern & High risk \\
\hline Makwana, 2001 & Low risk & Some concern & Some concern & Some concern & Some concern & High risk \\
\hline Marasco, 2013 & Low risk & Low risk & Low risk & Some concern & Some concern & $\begin{array}{l}\text { Some } \\
\text { concern }\end{array}$ \\
\hline $\begin{array}{l}\text { Matsunaga, } \\
2017\end{array}$ & Low risk & Some concern & Some concern & Some concern & Low risk & $\begin{array}{l}\text { Some } \\
\text { concern }\end{array}$ \\
\hline McKee, 2007 & Low risk & Low risk & Some concern & Some concern & Some concern & $\begin{array}{l}\text { Some } \\
\text { concern }\end{array}$ \\
\hline $\begin{array}{l}\text { Mirzatolooei, } \\
2011\end{array}$ & Low risk & Some concern & Some concern & Some concern & Some concern & High risk \\
\hline Mittal, 2017 & Low risk & Low risk & Some concern & Some concern & Low risk & $\begin{array}{l}\text { Some } \\
\text { concern }\end{array}$ \\
\hline Nouraei, 2011 & Some concern & Low risk & Some concern & Some concern & Some concern & High risk \\
\hline Olerud, $2011 \mathrm{a}$ & Low risk & Low risk & Low risk & Some concern & Some concern & $\begin{array}{l}\text { Some } \\
\text { concern }\end{array}$ \\
\hline
\end{tabular}


Table 2 Assessment of risk of bias of included trials of surgical and non-surgical treatment of fractures (Continued)

\begin{tabular}{|c|c|c|c|c|c|c|}
\hline Author, year & $\begin{array}{l}\text { Randomization } \\
\text { process }\end{array}$ & $\begin{array}{l}\text { Deviations from intended } \\
\text { interventions }\end{array}$ & $\begin{array}{l}\text { Missing } \\
\text { outcome data }\end{array}$ & $\begin{array}{l}\text { Measurement of the } \\
\text { outcome }\end{array}$ & $\begin{array}{l}\text { Selection of the } \\
\text { reported result }\end{array}$ & $\begin{array}{l}\text { Overall } \\
\text { bias }\end{array}$ \\
\hline Olerud, $2011 \mathrm{~b}$ & Low risk & Low risk & Low risk & Some concern & Some concern & $\begin{array}{l}\text { Some } \\
\text { concern }\end{array}$ \\
\hline Piazzolla, 2011 & Some concern & Low risk & Low risk & Some concern & Some concern & $\begin{array}{l}\text { Some } \\
\text { concern }\end{array}$ \\
\hline Qvist, 2018 & Low risk & Low risk & Some concern & Some concern & Low risk & $\begin{array}{l}\text { Some } \\
\text { concern }\end{array}$ \\
\hline Rangan, 2015 & Low risk & Some concern & Low risk & Some concern & Low risk & $\begin{array}{l}\text { Some } \\
\text { concern }\end{array}$ \\
\hline Robinson, 2013 & Low risk & Some concern & Low risk & Some concern & Some concern & $\begin{array}{l}\text { Some } \\
\text { concern }\end{array}$ \\
\hline Salai, 2000 & High risk & High risk & Some concern & Some concern & Some concern & High risk \\
\hline Sanders, 2012 & Low risk & Low risk & Low risk & Some concern & Some concern & $\begin{array}{l}\text { Some } \\
\text { concern }\end{array}$ \\
\hline Shen, 2001 & Some concern & High risk & Some concern & Some concern & Some concern & High risk \\
\hline Siebenga, 2006 & Some concern & Low risk & Low risk & Some concern & Some concern & $\begin{array}{l}\text { Some } \\
\text { concern }\end{array}$ \\
\hline Sletten, 2015 & Low risk & Low risk & Low risk & Some concern & Low risk & $\begin{array}{l}\text { Some } \\
\text { concern }\end{array}$ \\
\hline Smekal, 2009 & Low risk & Low risk & Low risk & Some concern & Some concern & $\begin{array}{l}\text { Some } \\
\text { concern }\end{array}$ \\
\hline Tamaoki, 2017 & Low risk & Low risk & Some concern & Some concern & Some concern & $\begin{array}{l}\text { Some } \\
\text { concern }\end{array}$ \\
\hline $\begin{array}{l}\text { Thordarson, } \\
1996\end{array}$ & Low risk & Low risk & Some concern & Some concern & Some concern & $\begin{array}{l}\text { Some } \\
\text { concern }\end{array}$ \\
\hline Vinnars, 2008 & Low risk & Low risk & Low risk & Some concern & Some concern & $\begin{array}{l}\text { Some } \\
\text { concern }\end{array}$ \\
\hline Virtanen, 2012 & Low risk & Low risk & Some concern & Some concern & Some concern & $\begin{array}{l}\text { Some } \\
\text { concern }\end{array}$ \\
\hline Willet, 2016 & Low risk & Low risk & Low risk & Some concern & Low risk & $\begin{array}{l}\text { Some } \\
\text { concern }\end{array}$ \\
\hline Woltz, 2017 & Low risk & Some concern & Some concern & Some concern & Low risk & $\begin{array}{l}\text { Some } \\
\text { concern }\end{array}$ \\
\hline Wong, 2010 & Low risk & Low risk & Some concern & Some concern & Some concern & $\begin{array}{l}\text { Some } \\
\text { concern }\end{array}$ \\
\hline Wood, 2003 & Low risk & Low risk & Some concern & Some concern & Some concern & $\begin{array}{l}\text { Some } \\
\text { concern }\end{array}$ \\
\hline Wu, 2018 & Low risk & Low risk & Low risk & Some concern & Some concern & $\begin{array}{l}\text { Some } \\
\text { concern }\end{array}$ \\
\hline Zyto, 1997 & Low risk & Low risk & Some concern & Some concern & Some concern & $\begin{array}{l}\text { Some } \\
\text { concern }\end{array}$ \\
\hline
\end{tabular}

Study quality was assessed for risk of bias using the Risk of Bias 2.0 tool from the Cochrane Collaboration on trials with results on patient-reported pain, physical function, and/or quality of life [14]. If four or five of the individual domains was found to be associated with some concerns of risk of bias, or if one of them was associated with high risk of bias, the overall risk of bias was rated as high risk

from surgical treatment is typically less than in younger more physically active patients. Thus, some of the studies included represent fracture types suspected to have limited benefits in terms of pain, function, and quality of life from surgical treatment. Other fracture types more obviously in need of surgery (displaced lower arm or hip fractures) is less likely to be subjected to randomization to non-surgical treatment; often termed parachute trials [87]. Despite the mentioned limitations of the SAE reporting, some interesting findings are worth mentioning as our study presents the first overview of SAEs across RCTs of different fractures. While the risk of SAEs was lower from surgical treatment in displaced midshaft clavicular fracture, it was higher in distal radius fractures, and no difference was present for the other six comparisons with the estimated relative risk of SAEs distributed relatively even on both sides of the "no 
difference in risk" line, dependent on the fracture type. Importantly, most of the findings were based on 2-3 studies, including few patients, precluding any firm conclusions. However, our results do suggest that for some of the more often studied fracture types, like displaced midshaft clavicular fractures, distal radius fractures in older patients, proximal humerus fractures, and traumatic thoraco-lumbar compression fractures, non-surgical treatment might serve as an equally effective and safe treatment as surgical treatment.

Only $20 \%$ of the most commonly performed orthopedic procedures, including surgery for fractures, are supported by at least one low risk of bias trial [88]. A search of trials of surgical and non-surgical treatment of fractures in the WHO International Clinical Trials Registry Platform [89] indicates that several ongoing trials will provide data to help build the evidence base for optimal treatment of fractures. Our study is a call to action for more low-risk-of-bias trials powered to detect any difference in benefits and harms between surgical and non-surgical treatment of the most common traumatic skeletal fractures in adults. Although such studies are known to be challenging [90], they are crucial to improve the clinical care of the patients.

\section{Conclusion}

Of 12 fracture types with data from more than one trial, only two demonstrated a difference in function in favor of surgery (moderate effect for displaced intraarticular calcaneal fractures, although affected by a large heterogeneity, and small effect for distal radial fractures), but with greater risk of harms after surgery for radial fractures. We found no difference in effect for displaced midshaft clavicular fractures, proximal humeral fractures, scaphoid waist, and thoracolumbar traumatic compression fractures, while surgery for clavicular fractures was associated with a reduced risk of SAE. Our results also highlight the current paucity of high-quality randomized trials for other common fracture types and a considerable heterogeneity for some of the estimates and risk of bias in a large proportion of available trials.

\section{Supplementary information}

Supplementary information accompanies this paper at https://doi.org/10. 1186/s13643-020-01424-4.

Additional file 1: S1. Search strategy for Medline. S2. Assessment of quality of harms assessment and reporting of included trials of surgical and non-surgical treatment of fractures. S3. Full forest plots for all comparisons, including deaths.

\section{Abbreviations}

SAE: Serious adverse event; BMI: Body mass index; SD: Standard deviation; SMD: Standardized mean difference; RR: Relative risk

\section{Acknowledgements}

The authors would like to thank MSc Mette Dideriksen for contributing to the submission of the manuscript.

\section{Authors' contributions}

Study conception and design. STS, CBJ, LSL, EMR. Acquisition of data. STS, CBJ. Analysis and interpretation of data. STS, CBJ, KBH, LSL, EMR. Drafting the article or revising it critically for important intellectual content. STS, CBJ, KBH, LSL, EMR. Final approval of the article. STS, CBJ, KBH, LSL, EMR. STS and CBJ had full access to all the data (including statistical reports and tables) in the study and take responsibility for the integrity of the data and the accuracy of the data analysis. The corresponding author attests that all listed authors meet authorship criteria and that no others meeting the criteria have been omitted and that the manuscript is an honest, accurate, and transparent account of the study being reported; that no important aspects of the study have been omitted; and that any discrepancies from the study as planned (and, if relevant, registered) have been explained. We plan to disseminate the results widely, including to patient organizations.

\section{Funding}

STS is currently funded by a grant from Region Zealand (Exercise First) and a grant from the European Research Council (ERC) under the European Union's Horizon 2020 research and innovation program (grant agreement No 801790). The study was partly funded by the Danish Physiotherapy

Association's fund for research, education and practice development.

\section{Availability of data and materials}

The datasets used and/or analyzed during the current study are available from the corresponding author on reasonable request.

Ethics approval and consent to participate

Not applicable

\section{Consent for publication}

Not applicable

\section{Competing interests}

EMR is deputy editor of Osteoarthritis and Cartilage, the developer of the Knee injury and Osteoarthritis Outcome Score (KOOS) and several other freely available patient-reported outcome measures and co-founder of Good Life with Osteoarthritis in Denmark (GLA:D), a not-for-profit initiative hosted at the University of Southern Denmark aimed at implementing clinical guidelines for osteoarthritis in clinical practice.

STS is an associate editor of the Journal of Orthopaedic \& Sports Physical Therapy, has received grants from The Lundbeck Foundation, personal fees from Munksgaard, all outside the submitted work. He is co-founder of GLA:D. GLA:D is a not-for-profit initiative hosted at the University of Southern Denmark aimed at implementing clinical guidelines for osteoarthritis in clinical practice.

The authors report no other conflict of interest.

\section{Author details}

'Research Unit for Musculoskeletal Function and Physiotherapy, Department of Sports Science and Clinical Biomechanics, University of Southern Denmark, Odense, Denmark. ${ }^{2}$ Department of Physiotherapy and Occupational Therapy, Næstved-Slagelse-Ringsted Hospitals, Region Zealand, Slagelse, Denmark. ${ }^{3}$ Department of Rehabilitation, Copenhagen University Hospital, Herlev and Gentofte, Denmark. ${ }^{4}$ Department of Orthopedics, Næstved-Slagelse-Ringsted Hospitals, Region Zealand, Slagelse, Denmark. ${ }^{5}$ Department of Regional Health Research, University of Southern Denmark, Odense, Denmark.

${ }^{6}$ Department of Clinical Sciences Lund, Orthopedics, Lund University, Lund, Sweden.

Received: 11 March 2020 Accepted: 10 July 2020

Published online: 13 August 2020

\section{References}

1. Donaldson LJ, Reckless IP, Scholes S, Mindell JS, Shelton NJ. The epidemiology of fractures in England. J Epidemiol Community Health. 2008; 62(2):174-80 Epub 2008/01/15. https://doi.org/10.1136/jech.2006.056622. PubMed PMID: 18192607. 
2. Curtis EM, van der Velde R, Moon RJ, van den Bergh JP, Geusens P, de Vries F, et al. Epidemiology of fractures in the United Kingdom 1988-2012: variation with age, sex, geography, ethnicity and socioeconomic status. Bone. 2016;87:19-26 Epub 2016/03/13. https://doi.org/10.1016/j.bone.2016. 03.006. PubMed PMID: 26968752; PubMed Central PMCID: PMCPMC4890652.

3. Court-Brown CM, Caesar B. Epidemiology of adult fractures: a review. Injury. 2006;37(8):691-7 Epub 2006/07/04. https://doi.org/10.1016/j.injury.2006.04. 130. PubMed PMID: 16814787.

4. Global Burden of Disease Study 2013 Collaborators. Global, regional, and national incidence, prevalence, and years lived with disability for 301 acute and chronic diseases and injuries in 188 countries, 1990-2013: a systematic analysis for the Global Burden of Disease Study 2013. Lancet. 2015; 386(9995):743-800 Epub 2015/06/13. https://doi.org/10.1016/s01406736(15)60692-4. PubMed PMID: 26063472; PubMed Central PMCID: PMCPMC4561509.

5. Leal J, Gray AM, Prieto-Alhambra D, Arden NK, Cooper C, Javaid MK, et al. Impact of hip fracture on hospital care costs: a population-based study. Osteoporos Int. 2016;27(2):549-58 Epub 2015/08/20. https://doi.org/10.1007/ s00198-015-3277-9. PubMed PMID: 26286626; PubMed Central PMCID: PMCPMC4740562.

6. Handoll $\mathrm{HH}$, Brorson S. Interventions for treating proximal humeral fractures in adults. Cochrane Database Syst Rev. 2015;(11):Cd000434 Epub 2015/11/ 13. https://doi.org/10.1002/14651858.CD000434.pub4. PubMed PMID: 26560014.

7. Handoll HH, Parker MJ. Conservative versus operative treatment for hip fractures in adults. Cochrane Database Syst Rev. 2008;(3):Cd000337 Epub 2008/07/23. https://doi.org/10.1002/14651858.CD000337.pub2. PubMed PMID: 18646065

8. Virtanen KJ, Malmivaara AO, Remes VM, Paavola MP. Operative and nonoperative treatment of clavicle fractures in adults. Acta Orthop. 2012 83(1):65-73 Epub 2012/01/18. https://doi.org/10.3109/17453674.2011. 652884. PubMed PMID: 22248169; PubMed Central PMCID: PMCPMC3278660.

9. Luo X, Li Q, He S, He S. Operative versus nonoperative treatment for displaced intra-articular calcaneal fractures: a meta-analysis of randomized controlled trials. J Foot Ankle Surg. 2016;55(4):821-8 Epub 2016/05/07. doi: https://doi.org/10.1053/j.jfas.2016.01.035. PubMed PMID: 27150233

10. Liberati A, Altman DG, Tetzlaff J, Mulrow C, Gotzsche PC, loannidis JP, et al. The PRISMA statement for reporting systematic reviews and meta-analyses of studies that evaluate healthcare interventions: explanation and elaboration. BMJ. 2009:339:b2700 Epub 2009/07/23. https://doi.org/10.1136/ bmj.b2700. PubMed PMID: 19622552; PubMed Central PMCID: PMCPMC2714672.

11. Higgins JPT, Green Se. Cochrane handbook for systematic reviews of interventions: the Cochrane collaboration; 2011. Available from: http:// handbook.cochrane.org.

12. Wartolowska K, Judge A, Hopewell S, Collins GS, Dean BJ, Rombach I, et al. Use of placebo controls in the evaluation of surgery: systematic review. BMJ (Clin Res Ed). 2014;348:g3253 https://doi.org/10.1136/bmi.g3253.

13. U. S. Food Drug Administration. What is a serious adverse event? Silver Spring, MD. 2014 [cited 201715 Feb]. Available from: http://www.fda.gov/ Safety/MedWatch/HowToReport/ucm053087.htm.

14. Higgins JPT, Sterne JAC, Savović J, Page MJ, Hróbjartsson A, Boutron I, et al. A revised tool for assessing risk of bias in randomized trials. In: Chandler J, McKenzie J, Boutron I, Welch V, editors. Cochrane Methods Cochrane Database of Systematic Reviews. Issue 10 (Suppl 1); 2016.

15. Chou R, Aronson N, Atkins D, Ismaila AS, Santaguida P, Smith DH, et al. Assessing harms when comparing medical interventions. Methods guide for effectiveness and comparative effectiveness reviews. Rockville (MD): Agency for Healthcare Research and Quality (US); 2008.

16. Cohen J. Statistical power analysis for the behavioral sciences. Hillsdale, NJ: Lawrence Earlbaum Associates; 1988.

17. Agren PH, Wretenberg P, Sayed-Noor AS. Operative versus nonoperative treatment of displaced intra-articular calcaneal fractures: a prospective, randomized, controlled multicenter trial. J BoneJoint Surg Ser A. 2013; 95(15):1351-7

18. Buckley R, Tough S, McCormack R, Pate G, Leighton R, Petrie D, et al. Operative compared with nonoperative treatment of displacedintra-articular calcaneal fractures - a prospective, randomized, controlled multicenter trial. J Bone Joint Surg-Am. 2002;84A(10):1733-44 https://doi.org/10.2106/ 00004623-200210000-00001. PubMed PMID: WOS:000178485600001.
19. O'Brien J, Buckley R, McCormack R, Pate G, Leighton R, Petrie D, et al. Personal gait satisfaction after displaced intraarticular calcaneal fractures: a 2-8 year followup. Foot Ankle Int. 2004;25(9):657-65. https://doi.org/10.1177/ 107110070402500911 Epub 2004/11/26. PubMed PMID: 15563389.

20. Griffin D, Parsons N, Shaw E, Kulikov Y, Hutchinson C, Thorogood M, et al. Operative versus non-operative treatment for closed, displaced, intraarticular fractures of the calcaneus: randomised controlled trial. BMJ (Online). 2014;349 Available from: http://onlinelibrary.wiley.com/o/cochrane/ clcentral/articles/183/CN-00999183/frame.html, http://www.bmj.com/ content/bmj/349/bmj.g4483.full.pdf.

21. Ibrahim T, Rowsell M, Rennie W, Brown AR, Taylor GJ, Gregg PJ. Displaced intra-articular calcaneal fractures: 15-year follow-up of a randomised controlled trial of conservative versus operative treatment. Injury. 2007;38(7): 848-55 Available from: http://onlinelibrary.wiley.com/o/cochrane/clcentral/ articles/576/CN-00609576/frame.html, http://ac.els-cdn.com/S00201383 07000046/1-s2.0-S0020138307000046-main.pdf?_tid=48c371 dc-2b41-11e79316-00000aabof6b\&acdnat=1493294685 735cad4ba57aa780a0a56 $808 f f e e 5683$.

22. Nouraei MH, Moosa FM. Operative compared to non-operative treatment of displaced intra-articular calcaneal fractures. J Res Med Sci. 2011;16(8):1014-9 PubMed PMID: 362576985.

23. Thordarson DB, Krieger LE. Operative vs. nonoperative treatment of intraarticular fractures of the calcaneus: a prospective randomized trial. Foot Ankle Int. 1996;17(1):2-9 Epub 1996/01/01. https://doi.org/10.1177/ 107110079601700102. PubMed PMID: 8821279.

24. Ahrens PM, Garlick NI, Barber J, Tims EM, Clavicle Trial Collaborative G. The clavicle trial: a multicenter randomized controlled trial comparing operative with nonoperative treatment of displaced midshaft clavicle fractures. J Bone Joint Surg-Am. 2017;99(16):10 https://doi.org/10.2106/jbjs.16.01112. PubMed PMID: WOS:000408218300008.

25. Chen QY, Kou DQ, Cheng XJ, Zhang W, Wang W, Lin ZQ, et al. Intramedullary nailing of clavicular midshaft fractures in adults using titanium elastic nail. Chin J Traumatol. 2011;14(5):269-76 PubMed PMID: CN01177374.

26. Judd DB, Pallis MP, Smith E, Bottoni CR. Acute operative stabilization versus nonoperative management of clavicle fractures. Am J Orthop (Belle Mead, NJ). 2009:38(7):341-5 Epub 2009/08/29. PubMed PMID: 19714275.

27. Koch HJ, Raschka C, Tonus C, Witzel K. The intramedullary osteosynthesis of the diaphyseal fracture of the clavicle compared to conservative treatment. Dtsch Z Sportmed. 2008;59(4):91-4 PubMed PMID: WOS:000256049600004.

28. Kumar A, Gulat DS. Conservative versus operative treatment of displaced mid shaft clavicle fracture - a two years randomized controlled study. J Evid Based Med Healthc. 2018;5(22):4.

29. McKee MD, Kreder HJ, Mandel S, McCormack R, Reindl R, Pugh DMW, et al. Nonoperative treatment compared with plate fixation of displaced midshaft clavicular fractures - a multicenter, randomized clinical trial. J Bone Joint Surg-Am. 2007;89A(1):1-10. https://doi.org/10.2106/jbjs.F.00020 PubMed PMID: WOS:000243270500001.

30. Melean PA, Zuniga A, Marsalli M, Fritis NA, Cook ER, Zilleruelo M, et al. Surgical treatment of displaced middle-third clavicular fractures: a prospective, randomized trial in a working compensation population. J Shoulder Elb Surg. 2015;24(4):587-92 Epub 2015/01/27. https://doi.org/10. 1016/j.jse.2014.11.041. PubMed PMID: 25619692.

31. Mirzatolooei F. Comparison between operative and nonoperative treatment methods in the management of comminuted fractures of the clavicle. Acta Orthop Traumatol Turc. 2011;45(1):34-40 Epub 2011/04/12. doi: https://doi. org/10.3944/aott.2011.2431. PubMed PMID: 21478660.

32. Qvist AH, Vaesel MT, Jensen CM, Jensen SL. Plate fixation compared with nonoperative treatment of displaced midshaft clavicular fractures: a randomized clinical trial. Bone Joint J. 2018;100-b(10):1385-91. https://doi. org/10.1302/0301-620x.100b10.Bij-2017-1137.R3 Epub 2018/10/09. PubMed PMID: 30295536

33. Robinson CM, Goudie EB, Murray IR, Jenkins PJ, Ahktar MA, Foster CJ, et al. Open reduction and plate fixation versus nonoperative treatment for displaced midshaft clavicular fractures. J BoneJoint Surg Ser A. 2013;95(17):1576-84.

34. Smekal V, Irenberger A, Struve P, Wambacher M, Krappinger D, Kralinger FS. Elastic stable intramedullary nailing versus nonoperative treatment of displaced midshaft clavicular fractures-a randomized, controlled, clinical trial. J Orthop Trauma. 2009;23(2):106-12 Epub 2009/01/27. https://doi.org/10. 1097/BOT.0b013e318190cf88. PubMed PMID: 19169102. 
35. Tamaoki MJS, Matsunaga FT, da Costa ARF, Netto NA, Matsumoto MH, Belloti JC. Treatment of displaced midshaft clavicle fractures: figure-of-eight harness versus anterior plate osteosynthesis a randomized controlled trial. J Bone Joint Surg-Am. 2017;99(14):1159-65 https://doi.org/10.2106/jbjs.16. 01184. PubMed PMID: WOS:000406759700005.

36. Virtanen KJ, Remes V, Pajarinen J, Savolainen V, Bjorkenheim JM, Paavola M. Sling compared with plate osteosynthesis for treatment of displaced midshaft clavicular fractures: a randomized clinical trial. J BoneJoint Surg Ser A. 2012;94(17):1546-53.

37. Woltz S, Stegeman SA, Krijnen P, van Dijkman BA, van Thiel TP, Schep NW, et al. Plate fixation compared with nonoperative treatment for displaced midshaft clavicular fractures: a multicenter randomized controlled trial. J Bone Joint Surg Am. 2017;99(2):106-12 Epub 2017/01/19. https://doi.org/10. 2106/jbjs.15.01394. PubMed PMID: 28099300.

38. Dugar N, Hossain E, Bandyopadhyay U, Shaw R. A comparative study of non-operative and operative management in fracture clavicle. J Indian Med Assoc. 2013;111(12):806, 8-9 PubMed PMID: CN-01014403.

39. Yadav $V$, Khare GN, Singh S, Kumaraswamy V, Sharma N, Rai AK, et al. A prospective study comparing conservative with operative treatment in patients with a 'floating shoulder' including assessment of the prognostic value of the glenopolar angle. Bone Joint J. 2013;95-B(6):815-9. https://doi. org/10.1302/0301-620X.95B6.31060 PubMed PMID: 2012138428. Language: English. Entry Date: 20130809. Revision Date: 20130809. Publication Type: journal article.

40. Chen ZW, Lin B, Zhai WL, Guo ZM, Liang Z, Zheng JP, et al. Conservative versus surgical management of Pipkin type I fractures associated with posterior dislocation of the hip: a randomised controlled trial. Int Orthop. 2011;35(7):1077-81 Available from: http://onlinelibrary.wiley.com/o/ cochrane/clcentral/articles/688/CN-00804688/frame.html, https://www.ncbi. nlm.nih.gov/pmc/articles/PMC3167412/pdf/264_2010_Article_1087.pdf.

41. Chen ZW, Zhai WL, Ding ZQ, Lian KJ, Kang LQ, Guo LX, et al. Operative versus nonoperative management of Pipkin type-Il fractures associated with posterior hip dislocation. Orthopedics. 2011;34(5):350 Available from: http://onlinelibrary. wiley.com/o/cochrane/clcentral/articles/861/CN-00799861/frame.html.

42. Matsunaga FT, Sugawara Tamaoki MJ, Hide Matsumoto M, Netto NA, Faloppa F, Belloti JC, et al. Minimally invasive osteosynthesis with a bridge plate versus a functional brace for humeral shaft fractures: a randomized controlled trial. J Bone Joint Surg Am. 2017;99(7):583-92. https://doi.org/10. 2106/JBJS.16.00628 PubMed PMID: 122353888. Language: English. Entry Date: 20171003. Revision Date: 20181030. Publication Type: journal article.

43. Boons HW, Goosen JH, Van Grinsven S, Van Susante JL, Van Loon CJ. Hemiarthroplasty for humeral four-part fractures for patients 65 years and older a randomized controlled trial. Clin Orthop Relat Res. 2012;470(12): 3483-91.

44. Fjalestad T, Hole M. Displaced proximal humeral fractures: operative versus non-operative treatment-a 2-year extension of a randomized controlled trial. Eur J Orthop Surg Traumatol. 2014;24(7):1067-73. https://doi.org/10.1007/ s00590-013-1403-y PubMed PMID: 103891640. Language: English. Entry Date: 20140922. Revision Date: 20151001. Publication Type: Journal Article.

45. Olerud P, Ahrengart L, Ponzer S, Saving J, Tidermark J. Internal fixation versus nonoperative treatment of displaced 3-part proximal humeral fractures in elderly patients: a randomized controlled trial. J Shoulder Elb Surg. 2011;20(5):747-55. https://doi.org/10.1016/j.jse.2010.12.018 Epub 2011/ 03/26. PubMed PMID: 21435907.

46. Olerud P, Ahrengart L, Ponzer S, Saving J, Tidermark J. Hemiarthroplasty versus nonoperative treatment of displaced 4-part proximal humeral fractures in elderly patients: a randomized controlled trial. J Shoulder Elb Surg. 2011;20(7):1025-33 Available from: http://onlinelibrary.wiley.com/o/ cochrane/clcentral/articles/532/CN-00810532/frame.html.

47. Rangan A, Handoll H, Brealey S, Jefferson L, Keding A, Martin BC, et al. Surgical vs nonsurgical treatment of adults with displaced fractures of the proximal humerus the PROFHER randomized clinical trial. JAMA. 2015;313(10 // 06/404/53 (HTA) *Health Technology Assessment Programme* // (NIHR) *Health Technology Assessment Programme*):1037-47 Available from: http://onlinelibrary.wiley.com/o/cochrane/clcentral/articles/653/CN-0106 9653/frame.html, http://jama.jamanetwork.com/pdfaccess.ashx?url=/data/ journals/jama/933474/joi150023.pdf.

48. Zyto K, Ahrengart L, Sperber A, Tornkvist H. Treatment of displaced proximal humeral fractures in elderly patients. J Bone Joint Surg Ser B. 1997;79(3):412-417. https://doi.org/10.1302/0301-620X.79B3.7419. PubMed PMID: 27232242.
49. Makwana NK, Bhowal B, Harper WM, Hui AW. Conservative versus operative treatment for displaced ankle fractures in patients over 55 years of age. J Bone Joint Surg Ser B. 2001;83(4):525-9 PubMed PMID: 32496378.

50. Willett K, Keene DJ, Mistry D, Nam J, Tutton E, Handley R, et al. Close contact casting vs surgery for initial treatment of unstable ankle fractures in older adults: a randomized clinical trial. Jama. 2016;316(14):1455-63 Epub 2016/10/12. https://doi.org/10.1001/jama.2016.14719. PubMed PMID: 27727383.

51. Mittal R, Harris IA, Adie S, Naylor JM. Surgery for Type B Ankle Fracture Treatment: a Combined Randomised and Observational Study (CROSSBAT). BMJ Open. 2017;7(3):e013298. https://doi.org/10.1136/bmjopen-2016-013298 Epub 2017/03/30. PubMed PMID: 28348185; PubMed Central PMCID: PMCPMC5372107.

52. Salai M, Dudkiewicz I, Novikov I, Amit Y, Chechick A. The epidemic of ankle fractures in the elderly - Is surgical treatment warranted? Arch Orthop Trauma Surg. 2000;120(9):511-3 https://doi.org/10.1007/s004020000172. PubMed PMID: 30706024.

53. Sanders DW, Tieszer C, Corbett B. Operative versus nonoperative treatment of unstable lateral malleolar fractures: a randomized multicenter trial. J Orthop Trauma. 2012;26(3):129-34 https://doi.org/10.1097/BOT. Ob013e3182460837. PubMed PMID: 364376911.

54. Sletten IN, Hellund JC, Olsen B, Clementsen S, Kvernmo HD, Nordsletten L. Conservative treatment has comparable outcome with bouquet pinning of little finger metacarpal neck fractures: a multicentre randomized controlled study of 85 patients. J Hand Surg Eur Vol. 2015;40(1):76-83. https://doi.org/ 10.1177/1753193414560119 Epub 2014/11/28. PubMed PMID: 25427553.

55. Strub B, Schindele S, Sonderegger J, Sproedt J, von Campe A, Gruenert JG. Intramedullary splinting or conservative treatment for displaced fractures of the little finger metacarpal neck? A prospective study. J Hand Surg Eur Vol. 2010;35(9):725-9. https://doi.org/10.1177/1753193410377845 Epub 2010/07/ 28. PubMed PMID: 20659966.

56. Lee TH, Lee JH, Chay SW, Jang KS, Kim HJ. Comparison of clinical and radiologic outcomes between non-operative and operative treatment in 5th metatarsal base fractures (Zone 1). Injury. 2016;47(8):1789-93 https://doi.org/ 10.1016/j.injury.2016.05.016. PubMed PMID: CN-01411345.

57. Wu GB, Li B, Yang YF. Comparative study of surgical and conservative treatments for fifth metatarsal base avulsion fractures (Type I) in young adults or athletes. J Orthop Surg (Hong Kong). 2018;26(1). https://doi.org/10. 1177/2309499017747128 PubMed PMID: CN-01643954.

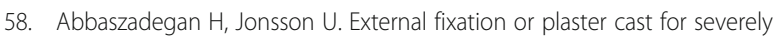
displaced Colles' fractures? Prospective 1-year study of 46 patients. Acta Orthop Scand. 1990;61(6):528-30 Epub 1990/12/01. PubMed PMID: 2281760

59. Arora R, Lutz M, Deml C, Krappinger D, Haug L, Gabl M. A prospective randomized trial comparing nonoperative treatment with volar locking plate fixation for displaced and unstable distal radial fractures in patients sixty-five years of age and older. J Bone Joint Surg Ser A. 2011;93(23):2146-53.

60. Azzopardi T, Ehrendorfer S, Coulton T, Abela M. Unstable extra-articular fractures of the distal radius. J Bone Joint Surg. 2005;87(6):837-40 Available from: http://onlinelibrary.wiley.com/o/cochrane/clcentral/articles/520/CN00569520/frame.html, http://www.bjj.boneandjoint.org.uk/content/jbjsbr/87B/6/837.full.pdf.

61. Földhazy Z, Ahrengart L. External fixation versus closed treatment of displaced distal radial fractures in elderly patients: a randomized controlled trial. Curr Orthop Pract. 2010;21(3):288-95 PubMed PMID: 105211643. Language: English. Entry Date: 20100709. Revision Date: 20150711. Publication Type: Journal Article.

62. Kreder HJ, Agel J, McKee MD, Schemitsch EH, Stephen D, Hanel DP. A randomized, controlled trial of distal radius fractures with metaphyseal displacement but without joint incongruity: closed reduction and casting versus closed reduction, spanning external fixation, and optional percutaneous K-wires. J Orthop Trauma. 2006;20(2):115-21 Available from: http://onlinelibrary.wiley.com/o/cochrane/clcentral/articles/500/CN-005625 00/frame.html, http://ovidsp.tx.ovid.com/ovftpdfs/FPDDNCDCLCHKAG00/ fs047/ovft/live/gv031/00005131/00005131-200602000-00006.pdf.

63. Stirrat GM, Farrow SC, Farndon J, Dwyer N. The challenge of evaluating surgical procedures. Ann R Coll Surg Engl. 1992;74(2):80-4 Epub 1992/03/01. PubMed PMID: 1567147; PubMed Central PMCID: PMCPMC2497523.

64. McQueen MM, Gelbke MK, Wakefield A, Will EM, Gaebler C. Percutaneous screw fixation versus conservative treatment for fractures of the waist of the scaphoid: a prospective randomised study. J Bone Joint Surg. 2008;90(1):66-71. https://doi.org/10.1302/0301-620X.90B1.19767 PubMed PMID: CN-00629042. 
65. Wong TC, Chiu Y, Tsang WL, Leung WY, Yam SK, Yeung SH. Casting versus percutaneous pinning for extra-articular fractures of the dista radius in an elderly Chinese population: a prospective randomised controlled trial. J Hand Surg. 2010;35(3):202-8 Available from: http:// onlinelibrary.wiley.com/o/cochrane/clcentral/articles/036/CN-00750036/ frame.html.

66. Marasco SF, Davies AR, Cooper J, Varma D, Bennett V, Nevill R, et al. Prospective randomized controlled trial of operative rib fixation in traumatic flail chest. J Am Coll Surg. 2013;216(5):924-32. https://doi.org/10.1016/j. jamcollsurg.2012.12.024 Epub 2013/02/19. PubMed PMID: 23415550.

67. Arora R, Gschwentner M, Krappinger D, Lutz M, Blauth M, Gabl M. Fixation of nondisplaced scaphoid fractures: making treatment cost effective. Prospective controlled trial. Arch Orthop Trauma Surg. 2007;127(1):39-46 Epub 2006/09/28. https://doi.org/10.1007/s00402-006-0229-z. PubMed PMID: 17004075.

68. Clementson M, Jorgsholm P, Besjakov J, Bjorkman A, Thomsen N. Union of scaphoid waist fractures assessed by CT scan. J Wrist Surg. 2015;4(1):49-55.

69. Dias JJ, Wildin CJ, Bhowal B, Thompson JR. Should acute scaphoid fractures be fixed? A randomized controlled trial. J BoneJoint Surg Ser A. 2005;87(10): 2160-8

70. Vinnars B, Pietreanu M, Bodestedt A, af Ekenstam F, Gerdin B. Nonoperative compared with operative treatment of acute scaphoid fractures. J Bone Joint Surg Am. 2008;90A(6):1176-85. https://doi.org/10.2106/jbjs.g.00673 PubMed PMID: WOS:000256456400002.

71. Piazzolla A, Giorgi S, Solarino G, Mori C, Giorgi G. Vertebral body reconstruction system B-Twin versus corset following non-osteoporotic Magerl A1.2 thoracic and lumbar fracture. Functional and radiological outcome at 12 month follow-up in a prospective randomized series of 50 patients. Orthop Traumatol Surg Res. 2011;97(8):846-51 Available from: http://onlinelibrary.wiley.com/o/cochrane/clcentral/articles/734/CN-00892 734/frame.html, http://ac.els-cdn.com/S1877056811002271/1-s2.0-\$1877056 811002271-main.pdf?_tid=d1b65978-2b41-11e7-81a9-00000aab0f2 7\&acdnat=1493294915_100b08468ed22eeb858f8a52e10f28a1.

72. Shen WJ, Liu TJ, Shen YS. Nonoperative treatment versus posterior fixation for thoracolumbar junction burst fractures without neurologic deficit. Spine. 2001;26(9):1038-45 Available from: http://onlinelibrary.wiley.com/o/ cochrane/clcentral/articles/694/CN-00347694/frame.html, http://ovidsp.tx. ovid.com/ovftpdfs/FPDDNCDCLCHKAG00/fs012/ovft/live/gv011/00007632/ 00007632-200105010-00010.pdf.

73. Siebenga J, Leferink VJ, Segers MJ, Elzinga MJ, Bakker FC, Haarman HJ, et al. Treatment of traumatic thoracolumbar spine fractures: a multicenter prospective randomized study of operative versus nonsurgical treatment. Spine. 2006;31(25):2881-90 Available from: http://onlinelibrary.wiley.com/o/ cochrane/clcentral/articles/110/CN-00574110/frame.html, http://ovidsp.tx. ovid.com/ovftpdfs/FPDDNCDCLCHKAG00/fs046/ovft/live/gv023/00007632/ 00007632-200612010-00003.pdf.

74. Wood K, Butterman G, Mehbod A, Garvey T, Jhanjee R, Sechriest V. Operative compared with nonoperative treatment of a thoracolumbar burst fracture without neurological deficit - A prospective, randomized study. J Bone Joint Surg Am. 2003;85A(5):773-81 PubMed PMID: WOS: 000182712100001.

75. Karladani AH, Granhed H, Edshage B, Jerre R, Styf J. Displaced tibial shaft fractures: a prospective randomized study of closed intramedullary nailing versus cast treatment in 53 patients. Acta Orthop Scand. 2000;71(2):160-7 Available from: http://onlinelibrary.wiley.com/o/cochrane/clcentral/articles/3 94/CN-00297394/frame.html.

76. Granetzny A, El-Aal MA, Emam E, Shalaby A, Boseila A. Surgical versus conservative treatment of flail chest. Evaluation of the pulmonary status. Interact Cardiovasc Thorac Surg. 2005;4(6):583-587. https://doi.org/10.1510/ icvts.2005.111807. PubMed PMID: 41744463

77. Hussain A, Nema SK, Sharma D, Akkilagunta S, Balaji G. Does operative fixation of isolated fractures of ulna shaft results in different outcomes than non-operative management by long arm cast? J Clin Orthop Traumatol. 2018;9(Suppl 1):S86-91 https://doi.org/10.1016/j.jcot.2017.12.004. PubMed PMID: CN-01440931

78. Duckworth AD, Clement ND, McEachan JE, White TO, Court-Brown CM, McQueen MM. Prospective randomised trial of nonoperative versus operative management of olecranon fractures in the elderly. Bone Joint J. 2017:99B(7):964-972. https://doi.org/10.1302/0301-620X.99B7.BJJ-2016-1112. R2. PubMed PMID: 617069242.
79. Clementson M, Jorgsholm P, Besjakov J, Thomsen N, Bjorkman A. Conservative treatment versus arthroscopic-assisted screw fixation of scaphoid waist fractures--a randomized trial with minimum 4-year followup. J Hand Surg. 2015;40(7):1341-8 Epub 2015/04/29. doi: https://doi.org/10. 1016/j.jhsa.2015.03.007. PubMed PMID: 25913660.

80. Mardani Kivi M, Asadi K, Hashemi Motlagh K, Shakiba M. Distal radius fracture, a comparison between closed reduction and long arm cast vs. closed reduction and percutaneous pinning and short arm cast. Shiraz $\mathrm{E}$ Med J. 2011;12(3):155-61 Available from: http://onlinelibrary.wiley.com/o/ cochrane/clcentral/articles/432/CN-00894432/frame.html.

81. Brorson S, Alispahic N, Bahrs C, Joeris A, Steinitz A, Audige L. Complications after non-surgical management of proximal humeral fractures: a systematic review of terms and definitions. BMC Musculoskelet Disord. 2019;20(1):91 https://doi.org/10.1186/s12891-019-2459-6 Epub 2019/02/25. PubMed PMID: 30797232; PubMed Central PMCID: PMCPMC6387518.

82. Checketts JX, Scott JT, Meyer C, Horn J, Jones J, Vassar M. The robustness of trials that guide evidence-based orthopaedic surgery. J Bone Joint Surg Am. 2018;100(12):e85 Epub 2018/06/20. https://doi.org/10.2106/jbjs.17.01039. PubMed PMID: 29916938.

83. Lochner HV, Bhandari M, Tornetta P 3rd. Type-Il error rates (beta errors) of randomized trials in orthopaedic trauma. J Bone Joint Surg Am. 2001;83(11): 1650-5 Epub 2001/11/10. https://doi.org/10.2106/00004623-20011100000005. PubMed PMID: 11701786.

84. Smeeing DPJ, van der Ven DJC, Hietbrink F, Timmers TK, van Heijl M, Kruyt $M C$, et al. Surgical versus nonsurgical treatment for midshaft clavicle fractures in patients aged 16 years and older: a systematic review, metaanalysis, and comparison of randomized controlled trials and observational studies. Am J Sports Med. 2017;45(8):1937-45 Epub 2016/11/20. doi: https:// doi.org/10.1177/0363546516673615. PubMed PMID: 27864184.

85. Bauer M, Jonsson K, Nilsson B. Thirty-year follow-up of ankle fractures. Acta Orthop Scand. 1985;56(2):103-6 Epub 1985/04/01. https://doi.org/10.3109/ 17453678508994329. PubMed PMID: 3925709

86. Kristensen KD, Hansen T. Closed treatment of ankle fractures. Stage II supination-eversion fractures followed for 20 years. Acta Orthop Scand. 1985:56(2):107-9 Epub 1985/04/01. https://doi.org/10.3109/ 17453678508994330. PubMed PMID: 3925710.

87. Katz JN. Parachutes and preferences--a trial of knee replacement. N Engl J Med. 2015;373(17):1668-9 Epub 2015/10/22. https://doi.org/10.1056/ NEJMe1510312. PubMed PMID: 26488698.

88. Lim HC, Adie S, Naylor JM, Harris IA. Randomised trial support for orthopaedic surgical procedures. PLoS One. 2014;9(6):e96745. https://doi. org/10.1371/journal.pone.0096745.

89. World Health Organization. WHO International Clinical Trials Registry Platform 2019. [cited 2019 Aug 16]. Available from: http://apps.who.int/ trialsearch/.

90. Cook JA. The challenges faced in the design, conduct and analysis of surgical randomised controlled trials. Trials. 2009;10:9 Epub 2009/02/10. https://doi.org/10.1186/1745-6215-10-9. PubMed PMID: 19200379; PubMed Central PMCID: PMCPMC2654883.

\section{Publisher's Note}

Springer Nature remains neutral with regard to jurisdictional claims in published maps and institutional affiliations.

Ready to submit your research? Choose BMC and benefit from:

- fast, convenient online submission

- thorough peer review by experienced researchers in your field

- rapid publication on acceptance

- support for research data, including large and complex data types

- gold Open Access which fosters wider collaboration and increased citations

- maximum visibility for your research: over $100 \mathrm{M}$ website views per year

At $\mathrm{BMC}$, research is always in progress.

Learn more biomedcentral.com/submission 